\title{
Suspension-based rheological modeling of crystallizing polymer melts
}

\author{
Rudi J. A. Steenbakkers • Gerrit W. M. Peters
}

Received: 10 July 2007 / Accepted: 20 February 2008 / Published online: 29 April 2008

(C) The Author(s) 2008

\begin{abstract}
The applicability of suspension models to polymer crystallization is discussed. Although direct numerical simulations of flowing particle-filled melts are useful for gaining understanding about the rheological phenomena involved, they are computationally expensive. A more coarse-grained suspension model, which can relate the parameters in a constitutive equation for the two-phase material to morphological features, such as the volume fractions of differently shaped crystallites and the rheological properties of both phases, will be more practical in numerical polymer processing simulations. General issues, concerning the modeling of linear and nonlinear viscoelastic phenomena induced by rigid and deformable particles, are discussed. A phenomenological extension of linear viscoelastic suspension models into the nonlinear regime is proposed. A number of linear viscoelastic models for deformable particles are discussed, focusing on their possibilities in the context of polymer crystallization. The predictions of the most suitable model are compared to direct numerical simulation results and experimental data.
\end{abstract}

Keywords Polymer crystallization • Structure development • Rheology • Suspension models

Paper presented at the 4th Annual European Rheology Conference (AERC), April 12-14, 2007, Naples, Italy.

R. J. A. Steenbakkers · G. W. M. Peters $(\bowtie)$

Department of Mechanical Engineering,

Eindhoven University of Technology (TU/e),

5600MB Eindhoven, The Netherlands

e-mail: g.w.m.peters@tue.nl

\section{Introduction}

The significant effects of flow on the crystallization kinetics of polymers, specifically the increase of the nucleation density and the transition from spherical to anisotropic growth, have incited a great deal of scientific effort, both experimental and theoretical. Experimental studies usually involve subjecting an undercooled melt to a short, well-defined flow in the early stage of crystallization, where nearly all of the material is still in the amorphous phase, and monitoring the subsequent structure development by any one of a variety of measurement techniques, including rheometry, microscopy, and scattering and diffraction methods, or a combination of these methods. Because our understanding of the phenomena occurring in the early stage, which determine to a great extent the final semicrystalline morphology, is still incomplete, it is not surprising that far less attention has been devoted to the influence of structure development on the rheology of a crystallizing melt. However, once the mechanisms of flow-induced crystallization are known, this will be the first step in going from short-term flow to continuous flow experiments, where the local process of phase transformation is affected by the development of semicrystalline structures on an orders of magnitude larger length scale and vice versa. These experiments will be useful as validation for polymer processing simulations.

During the last decade, a number of concepts have been proposed that deal with the rheology of crystallizing polymer melts. Winter and coworkers (Horst and Winter 2000a, b; Pogodina and Winter 1998; Pogodina et al. 1999a, b, 2001; Winter and Mours 1997) observed an apparent similarity to the rheology of chemical gels, in which polymer molecules are connected by 
permanent crosslinks into a sample spanning network. They considered crystallizing melts as physical gels, in which crystallites were connected by amorphous "tie chains."

Janeschitz-Kriegl et al. (2003) estimated the fraction of chains involved in nuclei in their experiments and found it to be so small that, during the major part of the crystallization process, no interaction among the nuclei or the resulting spherulites was to be expected. To explain the observed nonlinear increase of the nucleation density as a function of the mechanical work supplied to the melt, they introduced the concept of flow-induced activation of dormant nuclei (Janeschitz-Kriegl 2003; Janeschitz-Kriegl and Ratajski 2005).

Another explanation of the strong self-enhancing effect of nucleation was proposed by Zuidema (2000) and Zuidema et al. (2001). They assumed that nuclei locally act as physical crosslinks, increasing the probability that chain segments remain in an ordered state long enough to serve as new nuclei. In other words, gel-like behavior is not caused by the formation of a percolating network of semicrystalline domains but by effective branching of the amorphous phase. Lowfrequency rheological measurements, supporting this idea, were recently published by Coppola et al. (2006).

A few attempts have been made to capture the kinetics of flow-induced crystallization in a continuum description, embedded in a formal theoretical framework of nonequilibrium thermodynamics. For example, the Poisson bracket formalism (Beris and Edwards 1994) was used by Doufas et al. (1999). In their model, which was applied to flow-induced crystallization during fiber spinning (Doufas et al. 2000a, b; Doufas and McHugh 2001a, b) and film blowing (Doufas and McHugh 2001c), details of the microstructure, e.g., size and shape of the crystallites, are not taken into account. The crystalline phase is simply modeled as a collection of bead-rod chains. A Giesekus model is used for the amorphous phase, with the relaxation time depending on the degree of crystallinity $\chi$ as

$\lambda_{a m}=\lambda_{a m, 0}(1-\chi)^{2}$

to account for the loss of chain segments due to crystallization.

Hütter (2001) developed a flow-induced spherulitic crystallization model based on the "general equation for the nonequilibrium reversible-irreversible coupling” or GENERIC (Grmela and Öttinger 1997; Öttinger and Grmela 1997). The microstructure enters his model through the evolution of the interfacial area, obtained from the Schneider rate equations (Schneider et al. 1988). This gives rise to a pressure term in the mo- mentum balance, related to the surface tension, as well as to an interfacial heat flux in the energy balance. However, the extra stress tensor is written as the sum of the viscous stress contributions from the matrix and the spherulites,

$\boldsymbol{\tau}=\boldsymbol{\tau}_{a m}+\boldsymbol{\tau}_{s c}$,

as if the material were a homogenous mixture. Here, "am" stands for the amorphous matrix and "sc" for the partially crystalline, partially amorphous material inside the spherulites, which we call the semicrystalline phase. When both phases are incompressible, the partial stresses are given by

$\boldsymbol{\tau}_{a m}=2(1-\phi) \eta_{a m} \boldsymbol{D}$

and

$\boldsymbol{\tau}_{s c}=2 \phi \eta_{s c} \boldsymbol{D}$,

where $\phi$ is the volume fraction of spherulites, or degree of space filling, and $\boldsymbol{D}$ is the deformation rate tensor. Equations 2, 3, and 4 yield the effective viscosity

$\eta=(1-\phi) \eta_{a m}+\phi \eta_{s c}$.

Thus, no connection is made between rheological properties and microstructural features. Van Meerveld (2005) and Van Meerveld et al. (2008) extended Hütter's model with a description of the viscoelastic behavior of the melt and used it to simulate fiber spinning. In contrast to Hütter et al. (2005), who developed a single set of rate equations, allowing for changes in crystallite shapes and growth directions, they used two sets of rate equations to describe the evolution of spherulites and oriented crystallites. Although morphology development is incorporated in these models, at the continuum level, the stress is determined by the additive "rule of mixtures," Eq. 2. The question remains whether this is a realistic choice for describing the rheology of crystallizing polymer melts.

The morphology that develops as nuclei grow into crystallites with distinct shapes agrees with the basic concept of a suspension: isolated particles (the crystallites) are scattered throughout a continuous matrix (the amorphous phase). It is well known that the rule of mixtures fails to describe the volume fraction dependence of the rheological properties of suspensions. The same may hence be expected for crystallizing melts. Boutahar et al. (1996, 1998), Tanner $(2002,2003)$, and Van Ruth et al. (2006) therefore used ideas from suspension rheology to describe the evolution of linear viscoelastic properties during crystallization, as a function of the degree of space filling and the properties of the individual phases. 
Crystallizing polymer melts differ from ordinary suspensions in a number of ways. The crystallites grow, they can have different shapes depending on the flow history, and their properties evolve in time. The latter can be shown by combined optical microscopy and rheological measurements during crystallization. The dynamic modulus continues to increase after the completion of space filling (Van Ruth et al. 2006). This is the result of perfection of the semicrystalline phase, also referred to as secondary crystallization. In this paper, crystallites are therefore treated as particles whose properties depend on their internal degree of crystallinity,

$\chi_{1}=\frac{\chi}{\phi}$

thus providing the possibility to incorporate perfection in the model. The surrounding amorphous phase acts as a matrix, whose properties change as well. As yet unpublished results (J.F. Vega, personal communication) show strongly increased storage and loss moduli, measured at a constant frequency, directly after short steady shear flows. At the same time scale, no significant degree of space filling was observed by means of optical microscopy (D.G. Hristova, personal communication). Therefore, these results cannot be explained by particle-like effects of the crystallites on the overall rheology. Coppola et al. (2006) drew the same conclusion from a comparison of dynamic measurements on partially crystallized melts and on an amorphous melt filled with solid spheres. However, for the partially crystallized samples, the degrees of space filling were probably underestimated (see the "Frequency sweeps at different volume fractions" section).

To explain these observations, the amorphous matrix will be described as a crosslinking melt, with flowinduced nucleation precursors acting as physical crosslinks (Zuidema 2000; Zuidema et al. 2001). In the later stages of crystallization, the flow is severely disturbed by the presence of crystallites. Both phenomena have a nonlinear effect on the kinetics of flow-induced crystallization; furthermore, they are mutually coupled. The influence of flow on the early-stage kinetics, related to structure development within the amorphous matrix, will be discussed elsewhere. Two-dimensional (2D) simulations of flow-induced crystallization in a particlefilled polymer melt have already been performed without taking the physical crosslinking effect into account (Hwang et al. 2006). Here, we focus on the later stages of crystallization, which are dominated by space filling and perfection of the internal structure of the crystallites.
A suspension model for crystallization under real processing conditions has to meet at least the following requirements:

1. The model has to be applicable in the entire range of volume fractions, i.e., from the purely amorphous state $(\phi=0)$ to complete filling of the material by the crystallites $(\phi=1)$. This rules out dilute suspension theories, although an interpolation between analytical results for $\phi \rightarrow 0$ and $\phi \rightarrow 1$ has been applied with some success (Tanner 2003).

2. The possibility to incorporate differently shaped particles is essential for describing different semicrystalline morphologies. Here, spherulites and oriented crystallites are represented by spheres and cylinders, respectively, and we need a suspension model that can deal with both.

3. To describe the evolution of linear viscoelastic properties, as measured during crystallization, the model must provide a relationship between these properties and morphological features.

4. Quantitative description of most manufacturing processes requires that the effect of crystallization on the nonlinear viscoelastic behavior is captured as well.

In the "Linear viscoelastic suspension rheology" section, we briefly review how the effective dynamic mechanical properties of a linear viscoelastic suspension can be obtained from an elastic suspension model by means of the correspondence principle (Christensen 1969; Hashin 1965, 1970a, b). The consequences of modeling crystallites as either rigid or deformable particles are discussed. No specific suspension model is used; the discussion is of a general nature. A complementary phenomenological modeling approach to nonlinear viscoelastic suspension rheology is introduced in the "Nonlinear viscoelastic suspension rheology" section. Its ability to qualitatively reproduce results from experiments (Mall-Gleissle et al. 2002; Ohl and Gleissle 1993) and numerical simulations (Hwang et al. 2004a) is investigated. The properties of a specific linear viscoelastic suspension model are discussed in the "History and relation to other models" and "Influence of phase properties" sections. In the "Comparison to numerical and experimental data" section, its predictions are compared to numerical (Hwang et al. 2004a) and experimental (Mall-Gleissle et al. 2002) results for rigid particle suspensions. In the "Application to crystallization experiments" section, they are compared to experimental data on quiescent and short-term shear-induced crystallization of different polymer melts (J.F. Vega and D.G. Hristova, private communications; Boutahar 
et al. 1996, 1998; Coppola et al. 2006). The conclusions of this paper are summarized in the "Conclusions" section.

\section{Modeling}

Various constitutive models are available to describe the nonlinear viscoelastic behavior of the matrix of the suspension, i.e., the amorphous phase of the crystallizing melt. Differential models are most suited for numerical simulations of complex flows. Some of the most advanced are the Rolie-Poly model (Likhtman and Graham 2003) for linear melts and the Pom-Pom (McLeish and Larson 1998) and eXtended Pom-Pom (XPP: Verbeeten et al. 2004) models for branched melts. These and other differential models can be written in a general form, involving a slip tensor, which represents the nonaffine motion of polymer chains with respect to the macroscopic flow (Peters and Baaijens 1997).

The linear viscoelastic behavior of the matrix is characterized by the complex dynamic modulus, which is a function of the frequency $\omega$,

$G_{0}^{*}(\omega)=G_{0}^{\prime}(\omega)+j G_{0}^{\prime \prime}(\omega)$

and which is fitted by an $M$-mode discrete relaxation spectrum, giving the storage modulus

$G_{0}^{\prime}(\omega)=\sum_{i}^{M} G_{0, i} \frac{\lambda_{0, i}^{2} \omega^{2}}{1+\lambda_{0, i}^{2} \omega^{2}}$

and the loss modulus

$G_{0}^{\prime \prime}(\omega)=\sum_{i}^{M} G_{0, i} \frac{\lambda_{0, i} \omega}{1+\lambda_{0, i}^{2} \omega^{2}}$

in terms of the moduli $G_{0, i}$ and relaxation times $\lambda_{0, i}$. The influence of particles on the linear viscoelastic properties of a suspension is discussed next.

\section{Linear viscoelastic suspension rheology}

Our point of departure is the general expression for the effective shear modulus $G$ of a suspension of elastic particles dispersed throughout an elastic matrix (Torquato 2002),

$G(\phi)=f_{G}\left(\phi, s, \nu_{0}, \nu_{1}, \mu, \ldots\right) G_{0}$,

where $\phi$ is the volume fraction of the dispersed phase; $s$ is an array of shape factors that define the particle geometry; $v_{0}$ and $v_{1}$ are the Poisson ratios of the continuous phase and the dispersed phase, respectively; and $\mu$ is the ratio of the shear moduli of the phases,

$\mu=\frac{G_{1}}{G_{0}}$.

In general, $G_{0}$ and $G_{1}$ only occur in suspension models via this ratio. The dimensionless quantity $f_{G}=G / G_{0}$ is known as the relative shear modulus. Expressions analogous to Eq. 10 can be written down for the effective bulk modulus $K$, Young's modulus $E$, and Poisson ratio $v$ (Torquato 2002). Any two of these properties determine the mechanical behavior of an elastic material. In viscous systems, the relative viscosity $f_{\eta}=\eta / \eta_{0}$ is used.

To describe suspensions where both the matrix and the particles are linear viscoelastic, the effective dynamic shear modulus is written in the same form as in the elastic case,

$G^{*}(\omega, \phi)=f_{G}^{*}\left(\phi, \underset{\sim}{s}, \nu_{0}, \nu_{1}, \mu^{*}(\omega), \ldots\right) G_{0}^{*}(\omega)$

with $\mu^{*}=G_{1}^{*} / G_{0}^{*}$. This implies that $G_{0}^{*}$ and $G_{1}^{*}$ are known in the same range of frequencies. The relative dynamic shear modulus will later on be denoted by $f_{G}^{*}(\omega, \phi)$ or, simply, by $f_{G}^{*}$. However, one should keep in mind that, besides the frequency and the volume fraction, it also depends on the geometry of the particles and the material properties of the phases. The dynamic modulus ratio $\mu^{*}$ governs the frequency dependence of $f_{G}^{*}$, which makes it a complex quantity,

$f_{G}^{*}(\omega, \phi)=f_{G}^{\prime}(\omega, \phi)+j f_{G}^{\prime \prime}(\omega, \phi)$.

The Poisson ratios may, in principle, also be complex. However, experiments on different thermoplastic polymers have shown that the imaginary part of the complex Poisson ratio $v^{*}=v^{\prime}-j v^{\prime \prime}$ has a maximum at the glass transition temperature $T_{g}$, where it is about an order of magnitude smaller than the real part, i.e., $v^{\prime \prime} \sim$ $10^{-2}$, and that it decreases strongly upon departure from $T_{g}$ (Agbossou et al. 1993; Waterman 1977). We therefore assume that, in the present case, all Poisson ratios are real.

For a constant volume fraction, the correspondence principle (Christensen 1969; Hashin 1965, 1970a, b) relates the relative dynamic shear modulus $f_{G}^{*}$ to the relative shear modulus of an elastic suspension with the same microstructure. In the case of a steady-state oscillatory deformation with frequency $\omega, f_{G}^{*}$ is simply obtained by replacing the moduli $G_{0}$ and $G_{1}$ in the elastic model by their dynamic counterparts $G_{0}^{*}$ and $G_{1}^{*}$. Of course, the volume fraction of crystallites in a crystallizing polymer melt is not constant. However, according to Tanner (2003), if $\phi$ changes slowly compared to the characteristic time scale of stress relax- 
ation, the correspondence principle will still be a good approximation.

At this point, it should be noted that the density difference between the amorphous phase and the semicrystalline phase of a polymer has an influence on the volume fraction, which is given by

$\phi=\frac{\tilde{\phi} \rho_{a m}}{\tilde{\phi} \rho_{a m}+(1-\tilde{\phi}) \rho_{s c}}$.

Here, $\rho_{a m}$ and $\rho_{s c}$ are the densities of the amorphous and the semicrystalline phases, respectively. The volume fraction $\tilde{\phi}$, uncorrected for the density difference, is calculated as the volume of transformed amorphous phase per initial unit volume of material. Equation 14 can easily be included in the rate equations for the growth of the semicrystalline phase (Liedauer et al. 1993; Schneider et al. 1988). In the "Application to crystallization experiments" section, where the actual volume fraction is determined directly from microscopic images, no correction is necessary.

\section{Crystallites as rigid particles}

Because, in general, the dynamic modulus of a polymer increases by several orders of magnitude during crystallization, one may argue that the crystallites can be considered rigid. Any suspension model should make sure that, with this assumption, all occurrences of $\mu^{*}$ cancel each other out. This is trivial; if the infinite modulus ratio remained, the effective modulus of the suspension would already go to infinity when adding an infinitesimal amount of particles to the pure matrix, which is unrealistic. If the Poisson ratios are real, as we assume here, for rigid particles, the relative dynamic modulus thus becomes real as well,

$f_{G}(\phi) \equiv \lim _{\left|\mu^{*}\right| \rightarrow \infty} f_{G}^{*}\left(\phi, \mu^{*}(\omega)\right)$.

The effective storage modulus is then given by

$G^{\prime}(\omega, \phi)=f_{G}(\phi) \sum_{i}^{M} G_{0, i} \frac{\lambda_{0, i}^{2} \omega^{2}}{1+\lambda_{0, i}^{2} \omega^{2}}$

and the effective loss modulus by

$G^{\prime \prime}(\omega, \phi)=f_{G}(\phi) \sum_{i}^{M} G_{0, i} \frac{\lambda_{0, i} \omega}{1+\lambda_{0, i}^{2} \omega^{2}}$.

Hence, upon adding particles, all moduli increase by the same amount, which, moreover, is independent of the frequency, whereas the relaxation times remain equal to those of the matrix.
For suspensions in which the particles are essentially rigid, the validity of Eqs. 15, 16, and 17 has been confirmed by experiments, as well as numerical simulations. Schaink et al. (2000) investigated the individual effects of Brownian motion and hydrodynamic interactions on the viscosity of suspensions of rigid spheres by means of Stokesian dynamics simulations. They used a viscous fluid and a linear viscoelastic fluid as the matrix and found that the hydrodynamic contributions in both cases were similar. Expressions for the components $\eta^{\prime}=G^{\prime \prime} / \omega$ and $\eta^{\prime \prime}=G^{\prime} / \omega$ of the dynamic viscosity, equivalent to Eqs. 16 and 17, were obtained. Using the relative viscosity from the viscous simulation results, Schaink et al. were able to reproduce some of the oscillatory shear data of Aral and Kalyon (1997) for suspensions of glass spheres in a viscoelastic fluid, namely, those with $\phi=0.1$ and $\phi=0.2$. See et al. (2000) subjected suspensions of spherical polyethylene particles in two different viscoelastic matrix fluids to small-amplitude oscillatory squeezing flow. They found that, indeed, independent of the frequency, the relative quantities $\eta^{\prime}(\phi) / \eta_{0}^{\prime}$ of one system and $G^{\prime}(\phi) / G_{0}^{\prime}$ and $G^{\prime \prime}(\phi) / G_{0}^{\prime \prime}$ of the other system were all described by a single master curve in the examined volume fraction range, $0 \leq \phi \leq 0.4$.

Because we want to be able, in a later stage, to extend our work with a model for perfection of the semicrystalline phase and study its effect on mechanical properties, we prefer to treat crystallites as deformable particles. In this way, the possibility to model relatively weak (low $\chi_{1}$ ), as well as stiff (high $\chi_{1}$ ), semicrystalline structures also remains. Moreover, in numerical polymer processing simulations, it is preferable to work with a dynamic modulus that remains finite. This is not the case if crystallites are modeled as rigid particles up to large volume fractions.

Tanner (2003) proposed to use two separate models. The first gives $f_{G}$ for small volume fractions, assuming the crystallites to be rigid, according to Eq. 15. From the second model, which describes the crystallizing melt at large volume fractions, the additional relative dynamic modulus

$h_{G}^{*}=\frac{G^{*}}{G_{1}^{*}}$

is obtained. Depending on the microstructure of the system, we could, for example, use a model for densely packed particles, i.e., the crystallites, with the amorphous phase filling the interstices, or a suspension model with the amorphous phase as the particles and the semicrystalline phase as the matrix. In any case, the relevant dynamic modulus ratio is now $\mu^{*-1}$. It is 
assumed that the amorphous phase essentially consists of voids, so that

$h_{G}(1-\phi) \equiv \lim _{\left|\mu^{*}\right|^{-1} \rightarrow 0} h_{G}^{*}\left(1-\phi, \mu^{*-1}(\omega)\right)$.

An interpolation between the solutions of the smalland large-volume fraction models is necessary to insure a continuous transition at intermediate volume fractions. A linear interpolation has the general form

$G^{*}(\omega, \phi)=\mathcal{F}(\phi) G_{0}^{*}(\omega)+\mathcal{H}(\phi) G_{1}^{*}(\omega)$

with

$\mathcal{F}(\phi)=[1-w(\phi)] f_{G}(\phi)$

and

$\mathcal{H}(\phi)=w(\phi) h_{G}(\phi)$,

where $w \in[0,1]$ is an empirical weighting function. Tanner (2003) determined $\mathcal{F}$ and $\mathcal{H}$ directly, by fitting them to the oscillatory shear data of Boutahar et al. (1998) for a polypropylene melt containing different volume fractions of spherulites. A qualitative agreement with the shear-induced crystallization experiments of Wassner and Maier (2000) was found using these empirically determined interpolation functions. It should be noted that the experiments were limited to very low shear rates $(0.003 \leq \dot{\gamma} \leq 0.16)$.

If $G_{1}^{*}(\omega)$ is known and is fitted by a discrete relaxation spectrum of $N$ modes, Eqs. 16 and 17 are now extended to

$$
\begin{aligned}
G^{\prime}(\omega, \phi)= & \mathcal{F}(\phi) \sum_{i=1}^{M} G_{0, i} \frac{\lambda_{0, i}^{2} \omega^{2}}{1+\lambda_{0, i}^{2} \omega^{2}} \\
& +\mathcal{H}(\phi) \sum_{k=1}^{N} G_{1, k} \frac{\lambda_{1, k}^{2} \omega^{2}}{1+\lambda_{1, k}^{2} \omega^{2}}
\end{aligned}
$$

and

$$
\begin{aligned}
G^{\prime \prime}(\omega, \phi)= & \mathcal{F}(\phi) \sum_{i=1}^{M} G_{0, i} \frac{\lambda_{0, i} \omega}{1+\lambda_{0, i}^{2} \omega^{2}} \\
& +\mathcal{H}(\phi) \sum_{k=1}^{N} G_{1, k} \frac{\lambda_{1, k} \omega}{1+\lambda_{1, k}^{2} \omega^{2}} .
\end{aligned}
$$

In both the small-volume fraction model and the largevolume fraction model, all moduli change by the same amount while the relaxation times do not change. Due to the interpolation, however, the overall relaxation behavior of the material varies with the volume fraction, unless $M=N$ and $\lambda_{0, i}=\lambda_{1, i}$.

Although it is possible to capture, in this rather simple way, the evolution of linear viscoelastic properties during crystallization, we take a different approach. The linear viscoelastic modeling presented here will be extended to the nonlinear viscoelastic regime for application in polymer processing simulations. The interpolation method is not suited to this purpose because the optimal fitting parameters, defining the weighting function $w(\phi)$, probably change with the processing conditions.

\section{Crystallites as deformable particles}

In general, if $G_{1}^{*}$ is finite, $f_{G}^{*}$ is complex and Eq. 12 yields for the effective storage modulus

$G^{\prime}=\left(f_{G}^{\prime}-f_{G}^{\prime \prime} \tan \delta_{0}\right) G_{0}^{\prime}$

and for the effective loss modulus

$G^{\prime \prime}=\left(f_{G}^{\prime}+\frac{f_{G}^{\prime \prime}}{\tan \delta_{0}}\right) G_{0}^{\prime \prime}$

with $\tan \delta_{0}=G_{0}^{\prime \prime} / G_{0}^{\prime}$ tangent of the loss angle of the matrix. The fact that the expressions between parentheses in Eqs. 25 and 26 are different has an important consequence. Equation 25 can be written as

$G^{\prime}=\sum_{i=1}^{M}\left(f_{G}^{\prime}-\frac{f_{G}^{\prime \prime}}{\lambda_{0, i} \omega}\right) G_{0, i} \frac{\lambda_{0, i}^{2} \omega^{2}}{1+\lambda_{0, i}^{2} \omega^{2}}$

and Eq. 26 as

$G^{\prime \prime}=\sum_{i=1}^{M}\left(f_{G}^{\prime}+f_{G}^{\prime \prime} \lambda_{0, i} \omega\right) G_{0, i} \frac{\lambda_{0, i} \omega}{1+\lambda_{0, i}^{2} \omega^{2}}$.

It is clear that, if the effective relaxation times $\lambda_{i}$ are chosen equal to the relaxation times $\lambda_{0, i}$ of the matrix, $G^{\prime}$ and $G^{\prime \prime}$ can only be described by the same spectrum if $f_{G}^{\prime \prime}=0$. All moduli then increase by the same amount $f_{G}^{\prime}$ relative to those of the matrix, so that $G^{\prime}$ and $G^{\prime \prime}$ are shifted independent of the frequency, corresponding qualitatively to the behavior of a rigid particle suspension. However, $f_{G}^{\prime \prime}=0$ only if $\mu^{*}$ is real, i.e., if $G_{1}^{*}$ is proportional to $G_{0}^{*}$ so that both have the same frequency dependence, which is not the case in suspensions encountered in practice or in crystallizing polymer melts.

If $f_{G}^{\prime \prime} \neq 0, f_{G}^{*}$ must be determined in the whole range of frequencies of interest, given the dynamic moduli $G_{0}^{*}(\omega)$ and $G_{1}^{*}(\omega)$ of the individual phases. In numerical simulations of crystallization during flow, $G^{*}$ can, at any time step, be fitted by a new set of effective moduli and effective relaxation times, using the set from the previous time step as a first estimate. If the number of modes is the same for each phase, they can be expressed in terms of the moduli and relaxation times of the matrix as

$G_{i}(\phi)=k_{G, i}(\phi) G_{0, i}$ 
and

$\lambda_{i}(\phi)=k_{\lambda, i}(\phi) \lambda_{0, i}$

with $1 \leq k_{G, i} \leq G_{1, i} / G_{0, i}$ and $1 \leq k_{\lambda, i} \leq \lambda_{1, i} / \lambda_{0, i}$. In this way, a smooth transition from the matrix spectrum to the particle spectrum is obtained. If the latter consists of $N<M$ modes, while going from $\phi=0$ to $\phi=1, M-N$ of the initial $M$ modes should vanish. If $N>M, N-M$ new modes should appear. To ensure consistency, a single criterion must be used to choose the number of modes in the phase spectra and in the effective spectrum.

Thus, we use a single suspension model, in contrast to the interpolation method, where different models are used at small and large volume fractions. Therefore, we need a suspension model that is valid in the entire range of volume fractions, as stated in the Introduction. This severely limits the number of suitable models. We will come back to this in the "Evaluation of a linear viscoelastic model" section.

\section{Nonlinear viscoelastic suspension rheology}

The correspondence principle is only valid in the linear viscoelastic regime because it relies on the fact that the stress evolution is given by a Boltzmann integral (Christensen 1969; Hashin 1970a, b). In the context of modeling flow-induced crystallization during processing, nonlinear effects will be important at least in the amorphous phase, where the largest deformations take place. In general, nonlinear viscoelastic constitutive models contain the moduli $G_{i}$ and the relaxation times $\lambda_{i}$ of the linear relaxation spectrum plus a number of additional parameters. We assume that the correspondence principle still applies to the linear viscoelastic part of the rheology. The effective moduli and relaxation times are then related to those of the matrix by Eqs. 29 and 30, respectively.

Experiments on suspensions of rigid particles in a viscoelastic matrix have shown that the maximum strain amplitude, below which linear viscoelastic behavior is observed, decreases strongly with increasing particle volume fraction (Aral and Kalyon 1997). Thus, even though the matrix is linear viscoelastic, at a certain volume fraction, the behavior of the suspension will become nonlinear viscoelastic. This phenomenon may also be expected to occur if the particles are not rigid, although to our knowledge, no supporting data are available.

The experimental results of Ohl and Gleissle (1993) and Mall-Gleissle et al. (2002), in which suspensions of essentially rigid spheres in viscoelastic matrix fluids were subjected to simple shear flow, show a pronounced influence of the volume fraction on the normal stress differences. It was found that, for constant $\phi$, the steady-state first normal stress difference $N_{1}=\tau_{11}-\tau_{22}$ correlated with the shear stress as $N_{1} \sim$ $\tau_{12}^{n}$, where $1.63 \leq n \leq 1.68$. When the volume fraction of particles was increased at a constant value of the shear stress, they saw that the first normal stress difference decreased. This means that the dependence of $N_{1}$ on $\phi$ is weaker than that of $\tau_{12}^{n}$ on $\phi$.

Hwang et al. (2004a), who simulated 2D suspensions of rigid discs in an Oldroyd-B fluid, found a similar scaling of the time-averaged steady-state stress functions $N_{1}$ and $\tau_{12}$ with an exponent $n=2$. Furthermore, they showed that both the macroscopic shear viscosity $\eta=\tau_{12} / \dot{\gamma}_{0}$, where $\dot{\gamma}_{0}$ is the externally applied shear rate, and the macroscopic first normal stress coefficient $\Psi_{1}=N_{1} / \dot{\gamma}_{0}^{2}$ increase with $\dot{\gamma}_{0}$, as well as with $\phi$. MallGleissle et al. (2002) also observed that the magnitude of the second normal stress difference $\left|N_{2}\right|=\left|\tau_{22}-\tau_{33}\right|$ increased by the same amount as $N_{1}$ upon increasing the volume fraction at constant shear stress. This was not the case in the simulations of Hwang et al. (2004a) because the Oldroyd-B model does not predict a second normal stress difference in planar shear.

The dependence of $N_{1}$ and $\tau_{12}$ on the volume fraction of particles can be reproduced, at least qualitatively, by assuming that the "effective" velocity gradient tensor can be written as

$\boldsymbol{L}\left(\phi, \dot{\gamma}_{0}\right)=k_{L}\left(\phi, \dot{\gamma}_{0}\right) \boldsymbol{L}_{0}$

to take into account that the macroscopic velocity field $\boldsymbol{L}_{0}$ is locally disturbed by the presence of particles. The undisturbed shear rate, defined as

$\dot{\gamma}_{0}=\sqrt{2 \boldsymbol{D}_{0}: \boldsymbol{D}_{0}}$

with $\boldsymbol{D}_{0}=\frac{1}{2}\left[\boldsymbol{L}_{0}+\boldsymbol{L}_{0}^{T}\right]$ the undisturbed deformation rate tensor, together with the volume fraction of particles determines the strength of the disturbances $k_{L}$ according to Eq. 31. To illustrate this phenomenological model of particle-induced nonlinear effects, we choose a single-mode upper-convected Maxwell model. Using Eqs. 29, 30, and 31, the constitutive relation for the extra stress tensor becomes

$$
\stackrel{\nabla}{\tau}+\frac{1}{k_{\lambda} \lambda_{0}} \tau=2 k_{G} k_{L} G_{0} D_{0} .
$$

In a steady-state simple shear flow, Eq. 33 yields the shear stress

$\tau_{12}=k_{G} k_{\lambda} k_{L} G_{0} \lambda_{0} \dot{\gamma}_{0}$

and the first normal stress difference

$N_{1}=2 k_{G} k_{\lambda}^{2} k_{L}^{2} G_{0} \lambda_{0}^{2} \dot{\gamma}_{0}^{2}=\frac{2 \tau_{12}^{2}}{k_{G} G_{0}}$. 
In accordance with the numerical simulations of Hwang et al. (2004a), the first normal stress difference, at a given volume fraction, is proportional to the square of the shear stress. They used an Oldroyd-B model for the matrix, which leads to equivalent results if used in combination with the phenomenological nonlinear viscoelastic model discussed here. Furthermore, also in accordance with these simulations and with the experiments of Mall-Gleissle et al. (2002), the ratio of the first normal stress difference and the $n^{\text {th }}$ power (here $n=2$ ) of the shear stress, both normalized by their values at $\phi=0$, is independent of the shear stress and shear rate:

$\beta(\phi)=\frac{N_{1}\left(\phi, \dot{\gamma}_{0}\right) / N_{1}\left(\phi=0, \dot{\gamma}_{0}\right)}{\left[\tau_{12}\left(\phi, \dot{\gamma}_{0}\right) / \tau_{12}\left(\phi=0, \dot{\gamma}_{0}\right)\right]^{2}}=\frac{1}{k_{G}(\phi)}$.

Figure 1 shows how $\tau_{12}$ and $N_{1}$ change if the volume fraction is increased from $\phi_{1}$ to $\phi_{2}$ while the macroscopic shear rate is kept constant. For rigid particles $\left(k_{\lambda}=1\right)$ not disturbing the macroscopic velocity field $\left(k_{L}=1\right), N_{1}$ increases linearly with $\tau_{12}$. However, the results of Hwang et al. indicate that the dependence of $N_{1}$ on $\tau_{12}$, upon increasing the volume fraction at a constant shear rate, becomes stronger than linear. Here, this deviation is taken into account by the parameter $k_{L}$, which is a function of the volume fraction as well as the shear rate. Thus, with this parameter, a shear thickening is introduced, which is also in accordance with the simulations of Hwang et al. Moreover, it qualitatively agrees with the experiments of $\mathrm{Ohl}$ and Gleissle (1993) involving rigid particle suspensions with shear thinning matrix fluids, where the shear thinning effect was observed to decrease with increasing volume fraction at high shear rates.

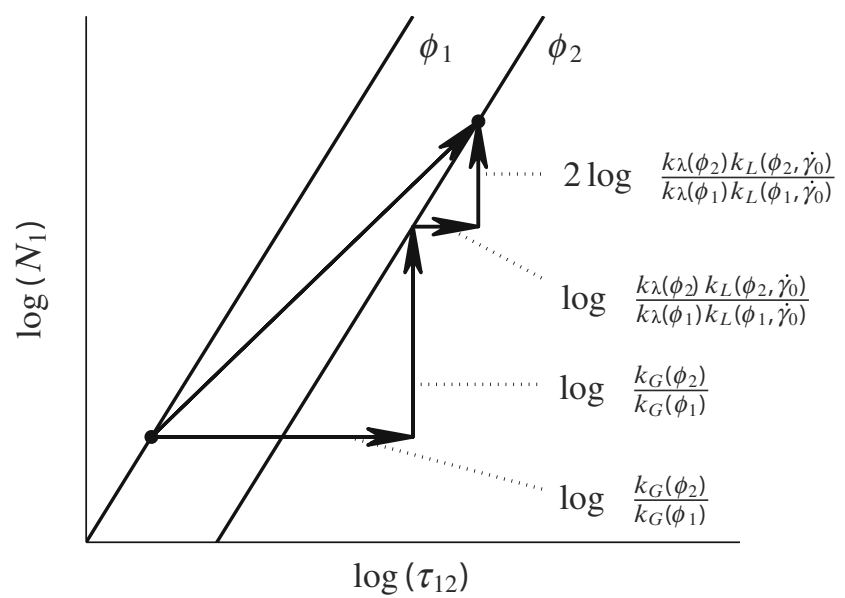

Fig. 1 The volume fraction dependence of the first normal stress difference and the shear stress at a macroscopic shear rate $\dot{\gamma}_{0}$
To describe the dependence of $N_{2}$ on $\phi$, a constitutive model should be chosen that predicts a negative second normal stress difference in simple shear flow. One option is to use a model with a GordonSchowalter derivative (Larson 1988) and a nonzero slip parameter $\zeta$, like the Phan-Thien-Tanner (PTT) model. However, it can be shown that $\beta$ then depends on the macroscopic Weissenberg number, $\mathrm{Wi}_{0}=$ $\lambda_{0} \dot{\gamma}_{0}$, which contradicts the experimental results. Via a different approach, Tanner and Qi (2005) developed a phenomenological nonlinear viscoelastic suspension model, showing reasonable agreement with experimental data for $N_{1}$ and $N_{2}$. The stress tensor in their model consists of two modes. One is described by a PTT model, with $\zeta=0$ and including a volume fraction dependence of the relaxation time, and the other by a Reiner-Rivlin model with a volume fraction-dependent viscosity. The latter causes the second normal stress difference. A definitive validation of the method proposed here may be possible by starting with more advanced constitutive models, like, for example, the Rolie-Poly (Likhtman and Graham 2003), Pom-Pom (McLeish and Larson 1998), or XPP (Verbeeten et al. 2004) models.

As shown in Fig. 1, shifting the shear stress and the first normal stress difference by $k_{G}(\phi)$, we should end up on the line with slope $n$ corresponding to the volume fraction $\phi$. Hence, experimental data such as those of Mall-Gleissle et al. can be used to validate any combination of a constitutive model for the matrix and a suspension model for the influence of the particles on the effective linear viscoelastic properties. Moreover, numerical results like those of Hwang et al. allow for the independent validation of linear viscoelastic models for suspensions of rigid particles because the same constitutive model for the matrix can be chosen as in the simulations. Unfortunately, we are not aware of similar experimental or numerical results for deformable particles.

The parameter $k_{L}$, which describes the nonlinear viscoelasticity induced by the presence of particles, can be found by fitting it to experimental or numerical data. This procedure is not independent of the constitutive model used for the matrix because different constitutive models may yield different values for the exponent $n$. For the upper-convected Maxwell and Oldroyd-B models, where $n=2$, we find

$k_{L}\left(\phi, \dot{\gamma}_{0}\right)=\frac{N_{1}\left(\phi, \dot{\gamma}_{0}\right) / N_{1}\left(\phi=0, \dot{\gamma}_{0}\right)}{\tau_{12}\left(\phi, \dot{\gamma}_{0}\right) / \tau_{12}\left(\phi=0, \dot{\gamma}_{0}\right)}$.

Figure 1 shows that this parameter determines the deviation from the curve $N_{1} \sim \tau_{12}$, when the volume fraction is increased at a constant macroscopic shear rate $\dot{\gamma}_{0}$. Hwang et al. showed the dependence of $k_{L}$ on the 
volume fraction and the macroscopic shear rate in their 2D simulations (Fig. 8 in Hwang et al. 2004a).

In the following, an elastic suspension model, taken from the literature, is transformed to a linear viscoelastic model by means of the correspondence principle. Its predictions are compared qualitatively to the numerical simulations of Hwang et al. and the experiments of Mall-Gleissle et al. in the "Comparison to numerical and experimental data" section and quantitatively to crystallization experiments in the "Application to crystallization experiments" section. A quantitative evaluation of the phenomenological model of nonlinear viscoelastic suspension rheology, discussed above, is beyond the scope of this paper.

\section{Evaluation of a linear viscoelastic model}

Analytical descriptions of the effects of particles on the rheology of a suspension are generally restricted to isolated particles or to interactions between pairs of particles and are, therefore, valid only in dilute or semidilute conditions, respectively. In the case of a crystallizing polymer melt, however, we need a suspension model that is applicable in the entire range of volume fractions. An appropriate choice might be one of the socalled self-consistent estimates, which have been used for quite some time in the mechanical modeling of elastic composites. Essentially, the effective properties are found as follows: A stress or strain is prescribed at the boundary of a unit cell, which gives a simplified picture of the microstructure. The mechanical response of the unit cell is calculated, and when this response becomes homogeneous, the effective mechanical properties are found.

The generalized self-consistent method of Christensen and Lo (1979) was claimed by these authors to be valid in the entire range of volume fractions. Furthermore, it gives solutions for suspensions of spherical particles and suspensions of long parallel cylindrical fibers, corresponding to the different microstructures found locally in a crystallizing polymer melt. The generalized self-consistent method thus meets the first two requirements stated in the Introduction. The third and fourth have already been dealt with in the "Modeling" section. We therefore discuss this model in detail here.

\section{History and relation to other models}

The generalized self-consistent method was originally called three-phase model, but it was renamed by Christensen (1990) in reference to the self-consistent method (Hill 1965a, b; Budiansky 1965). This model, which has an analogy in the theory of heterogenous conducting materials (Bruggeman 1935), considers a single particle embedded in a homogeneous matrix with the effective properties sought. The generalized self-consistent method, on the other hand, uses a unit cell made up of a particle surrounded by a concentric shell of the matrix material. This coated particle is embedded in the effective homogeneous medium. The difference between the two models can be interpreted as follows: "While the self-consistent method seeks to predict the interaction of an inclusion and its neighboring microstructure (the combined effect of the matrix and other inclusions), this model includes (in a certain approximate sense) the interaction between the inclusion and the surrounding matrix, as well as the neighboring microstructure" (Nemat-Nasser and Hori 1993). A coated particle unit cell was used earlier by Fröhlich and Sack (1946) for elastic spheres in a viscous matrix, by Oldroyd (1953) for viscous drops or elastic spheres in a viscous matrix, by Kerner (1956) for elastic spheres in an elastic matrix, and by Hermans (1967) for unidirectional elastic fibers in an elastic matrix. Two versions of the generalized self-consistent method exist: a three-dimensional (3D) one in which the particle and matrix domains of the unit cell are concentric spheres and a 2D one in which they are concentric circles. These give the solutions for spherical particles and long parallel cylindrical fibers, respectively.

Table 1 summarizes the main properties of the generalized self-consistent method and some other suspension models. Palierne (1990) developed a model for incompressible linear viscoelastic emulsions in which the drops are at least approximately spherical. For dilute emulsions, he considered a single drop suspended in the effective medium. Not surprisingly, neglecting the effect of surface tension, the result is the same as the analytical solution of the self-consistent method in the dilute limit, taking the matrix as incompressible (Hill 1965b). In contrast to both the self-consistent and the generalized self-consistent methods, the derivation of the nondilute Palierne model is based on a unit cell in which one particle is at the center of a sphere filled with the matrix and other particles, which in turn is surrounded by the effective medium. If the effect of surface tension is again neglected, it turns out that the result is exactly the viscoelastic analogue of the model of Kerner (1956) for all volume fractions (Graebling et al. 1993; Palierne 1990). Through a similar derivation for an elastic suspension of spheres, Uemura and Takayanagi (1966) also arrived at the same effective shear modulus as Kerner, although a different expression for the effective Poisson ratio was obtained. 
Table 1 Attributes of some suspension models

\begin{tabular}{|c|c|c|c|c|}
\hline Model & Phases & Particles & Volume fraction & Ref. \\
\hline SCM & & $\begin{array}{l}\text { Spheres } \\
\text { Cylinders }\end{array}$ & Small or large & $\begin{array}{l}\text { Hill (1965b) } \\
\text { Hill (1965a) }\end{array}$ \\
\hline GSCM & $\mathrm{E}$ & $\begin{array}{l}\text { Spheres } \\
\text { Cylinders }\end{array}$ & $\begin{array}{l}\text { Arbitrary, size distribution } \\
\text { should admit } \phi \rightarrow 1\end{array}$ & Christensen and Lo $(1979,1986)$, Christensen (1990) \\
\hline $\begin{array}{l}\text { Torquato, exact } \\
\text { Torquato, TOA }\end{array}$ & & Arbitrary & $\begin{array}{l}\text { Arbitrary } \\
\text { Small to moderate }\end{array}$ & $\begin{array}{l}\text { Torquato (1997) } \\
\text { Torquato (1998) }\end{array}$ \\
\hline $\begin{array}{l}\text { Palierne } \\
\text { Bousmina }\end{array}$ & LVE & Spheres & Small to moderate & $\begin{array}{l}\text { Palierne (1990) } \\
\text { Bousmina (1999) }\end{array}$ \\
\hline
\end{tabular}

$S C M$ self-consistent method, GSCM generalized self-consistent method, TOA third-order approximation, $E$ elastic, $L V E$ linear viscoelastic

Christensen and Lo (1979) demonstrated that the elastic shear modulus predicted by the 3D generalized self-consistent method lies between the classical upper (Hashin 1962) and lower bounds (Hashin and Shtrikman 1963; Walpole 1966) for all volume fractions, whereas Kerner's result coincides with the lower bound. Contrary to Palierne's model, the 3D generalized self-consistent method is only equivalent to Kerner's model for vanishing volume fraction of spheres. Whereas Palierne did not consider drops close to contact with each other, Christensen (1990) derived, by physical reasoning similar to that of Frankel and Acrivos (1967), the functional form of $f_{G}(\phi)$ and of the relative transverse shear modulus $f_{G_{23}}(\phi)$ for rigid spheres and unidirectional rigid cylinders, respectively, when $\phi \rightarrow 1$. They were found to agree with the corresponding asymptotic forms of the generalized selfconsistent method, for a compressible matrix and for an incompressible matrix. Of course, the volume fraction can only go to one if the distribution of particle diameters is sufficiently broad, so that small particles can fill the spaces between larger particles.

Bousmina (1999) proposed an emulsion model based on the 3D generalized self-consistent method, extending the particle modulus with a term due to surface tension. In the coefficients of the quadratic function, one of whose roots is $f_{G}^{*}$ (see Eq. 41 later on), only terms of order $\phi$ were retained. It is therefore not surprising that only small differences with Palierne's model were observed. The expressions for the coefficients given in Bousmina's paper contain a few errors, apparently mostly because he was unaware of an erratum (Christensen and Lo 1986) to the original paper on the generalized self-consistent method; the correct expressions are included here in the Appendix.

Christensen (1990) validated the 3D generalized self-consistent method with respect to experimental data on suspensions of rigid spheres. The results proved superior to those of two homogenization schemes widely used at that time, i.e., the Mori-Tanaka method (Benveniste 1987) and the differential scheme (PhanThien and Pham 1997), especially for volume fractions $\phi>0.4$. However, Nemat-Nasser and Yu (1993) pointed out uncertainties in some of the experimental data used for comparison, which were compiled by Thomas (1965). Segurado and Llorca (2002) performed $3 \mathrm{D}$ numerical simulations of suspensions of spheres in an elastic matrix, where $0 \leq \phi \leq 0.5$. They compared their results to the predictions of the Mori-Tanaka method, the generalized self-consistent method, and the third-order approximation (Torquato 1998) of an exact series expansion for the effective stiffness tensor of elastic two-phase media (Torquato 1997). The generalized self-consistent method performed just as well as this third-order approximation when the particles were deformable, except that the effective bulk modulus was predicted slightly more accurately by the third-order approximation. For rigid spheres, the latter also yielded somewhat better results.

Because the generalized self-consistent method is much easier to implement than Torquato's third-order approximation, we will use it to determine the linear viscoelastic properties of a suspension with the aid of the correspondence principle. As explained above, the suspension is represented by a unit cell consisting of a particle and a surrounding matrix shell. Their radii are $a$ and $b$, respectively. The volume fraction of particles is given by

$\phi=\left(\frac{a}{b}\right)^{3}$

for the 3D coated sphere unit cell and

$\phi=\left(\frac{a}{b}\right)^{2}$ 
for the 2D coated cylinder unit cell. The unit cell is suspended in an infinitely extending effective medium, which has the effective properties of the suspension. These properties are found when the response of the unit cell to a given load equals the response of the homogeneous effective medium. In the 3D generalized self-consistent method, the relative shear modulus (see Eq. 10) is obtained from the quadratic equation

$A f_{G}^{2}+B f_{G}+C=0$

where the coefficients $A, B$, and $C$ depend on $\phi, \mu, v_{0}$, and $v_{1}$. These coefficients are given in the Appendix. The relative bulk modulus was found to be the same as in the composite spheres model of Hashin (1962).

For elastic suspensions of long parallel cylindrical fibers, Hashin and Rosen (1964) derived the components of the fourth-order stiffness tensor, except the shear modulus in the transverse plane. The 2D generalized self-consistent method gives the relative transverse shear modulus as the solution of a quadratic expression similar to Eq. 40, but with different coefficients. These are also included in the Appendix.

In accordance with the correspondence principle, the relative dynamic modulus is obtained from

$A^{*} f_{G}^{* 2}+B^{*} f_{G}^{*}+C^{*}=0$,

where the complex coefficients $A^{*}, B^{*}$, and $C^{*}$ follow from $A, B$, and $C$ when $\mu$ is replaced by $\mu^{*}$. For a crystallizing polymer melt, we propose to calculate the effect of the presence of spherulites by means of the 3D generalized self-consistent method and to use the resulting effective medium as the matrix in the $2 \mathrm{D}$

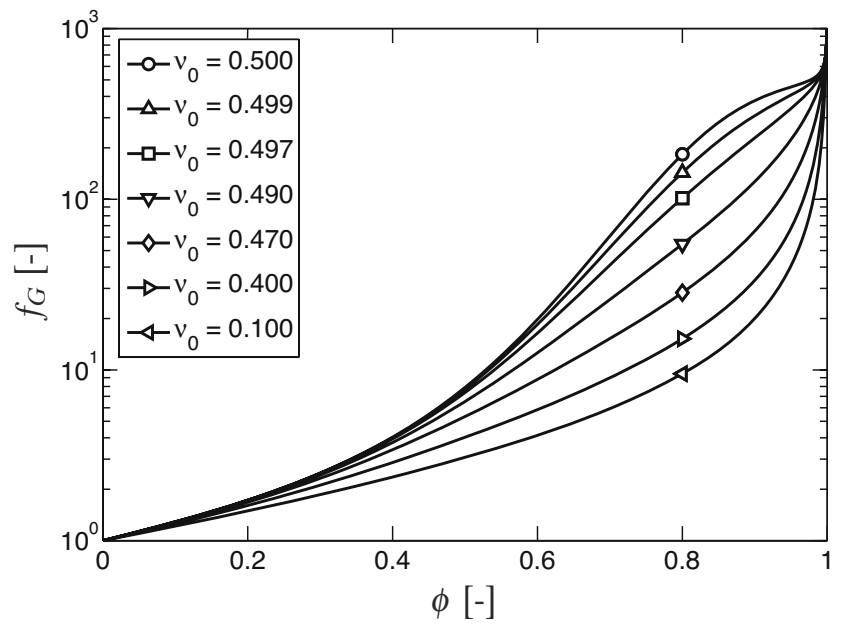

Fig. 2 Influence of the Poisson ratio of the matrix on the relative modulus of an elastic suspension of spheres $\left(\mu=10^{3}, v_{1}=0.5\right)$

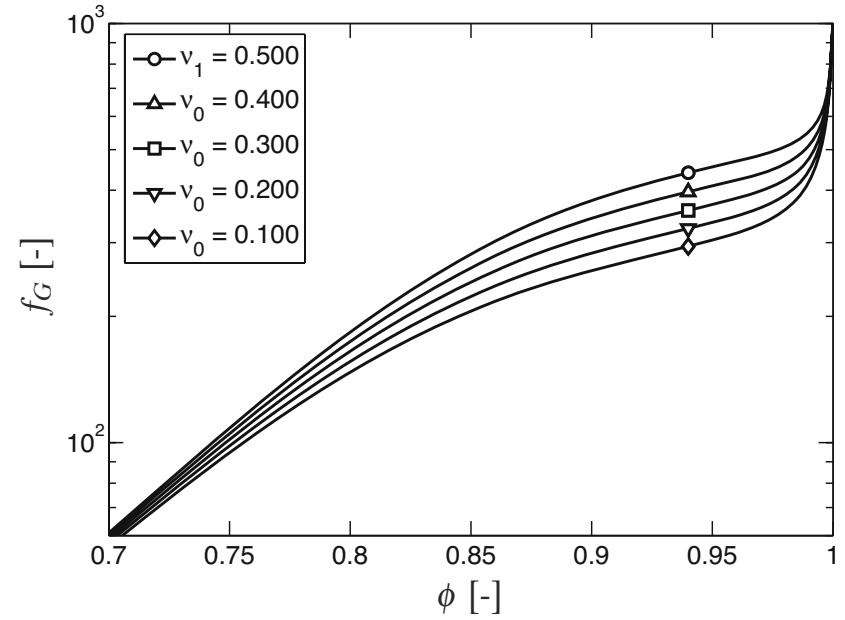

Fig. 3 Influence of the Poisson ratio of the particles on the relative modulus of an elastic suspension of spheres $\left(\mu=10^{3}\right.$, $\left.v_{0}=0.5\right)$

generalized self-consistent method, which accounts for the influence of oriented crystallites.

Influence of phase properties

First of all, let us take a look at the original 3D generalized self-consistent method for elastic suspensions of spheres, according to which the relative shear modulus $f_{G}$ is obtained from Eq. 40 with real coefficients $A$, $B$, and $C$. The curve of $\log \left(f_{G}\right)$ vs $\phi$ for $\mu=10^{3}$ and $v_{0}=v_{1}=0.5$, plotted in Figs. 2 and 3, has two inflection points: one at $\phi \approx 0.70$ and the other at $\phi \approx 0.95$. In between these points, the second derivative is negative,

$\frac{\partial^{2} \log \left(f_{G}\right)}{\partial \phi^{2}}<0$,

and consequently, a "shoulder" appears in the curve. Beyond $\phi \approx 0.95, f_{G}$ swiftly approaches its final value $f_{G}(\phi=1)=\mu$; note that $f_{G}$ at $\phi=0.95$ is still smaller than $\mu / 2$.

The shape of the relative modulus curve depends most strongly on the Poisson ratio of the matrix and on the modulus ratio. Figure 2 shows that, upon lowering $v_{0}$ while keeping $v_{1}=0.5, f_{G}$ decreases and the shoulder vanishes quickly: at $v_{0}=0.49$, it is not recognizable anymore. Decreasing $v_{1}$ while $v_{0}=0.5$ has a much weaker influence on $f_{G}$, as seen in Fig. 3, and the shoulder remains. Thus, even at large volume fractions, compressibility of the matrix has a more profound influence on the results of the 3D generalized selfconsistent method than compressibility of the particles. Furthermore, the shoulder diminishes at lower values of the modulus ratio, as shown in Fig. 4. It should be noted that the logarithmic scale used on the vertical 


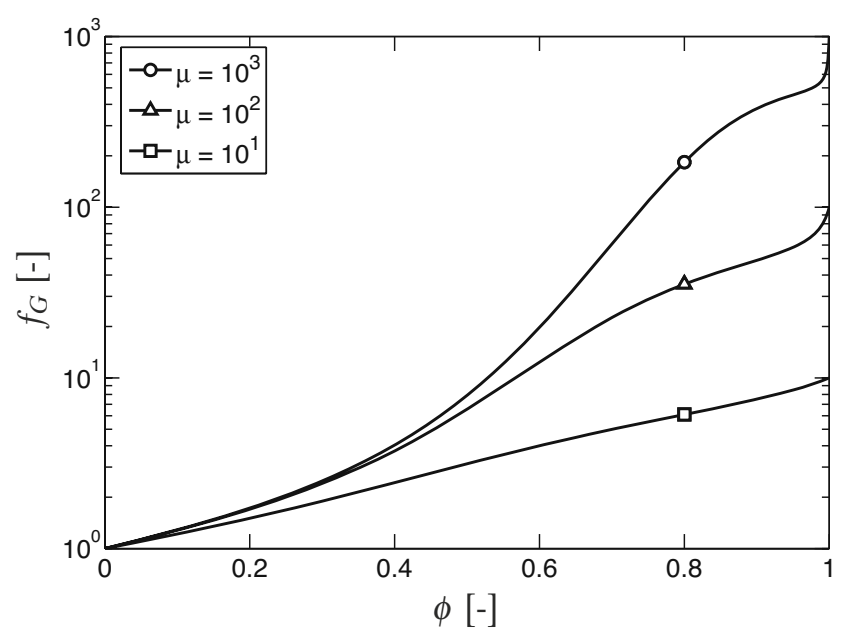

Fig. 4 Influence of the modulus ratio on the relative modulus of an elastic suspension of spheres $\left(v_{0}=v_{1}=0.5\right)$

axes of these figures exaggerates the effects mentioned. For the second derivative of $f_{G}$ with respect to $\phi$, one finds

$\frac{\partial^{2} f_{G}}{\partial \phi^{2}}<\frac{1}{f_{G}}\left(\frac{\partial f_{G}}{\partial \phi}\right)^{2}$

if Eq. 42 is satisfied. Because the right-hand side of Eq. 43 is always positive, when $f_{G}$ is plotted on a linear scale, a smaller decrease of $v_{0}$ or $\mu$ suffices to make the shoulder vanish: it is already gone at a matrix Poisson ratio $v_{0}=0.498$ for $v_{1}=0.5$ and $\mu=10^{3}$, and at a modulus ratio $\mu=50$ for $v_{0}=v_{1}=0.5$.

To our knowledge, this peculiar feature of the generalized self-consistent method has never been reported before. Looking at the material parameters in previous publications, it is easy to see why. Christensen and Lo (1979) simulated suspensions with modulus ratios $\mu=23.46$ and $\mu=135.14$. According to Fig. 4, the latter is high enough to cause an observable shoulder in a suspension with an incompressible matrix, but Christensen and Lo used a matrix with $v_{0}=0.35$, which is too low even when $\mu=10^{3}$ (Fig. 2). In the later work of Christensen (1990), the compressibility effect was obscured because only rigid particles were considered $(\mu \rightarrow \infty)$. The high end of the curve is then stretched to infinity, so that the shoulder is smoothed out. Segurado and Llorca (2002) used the 3D generalized self-consistent method to simulate both suspensions of rigid spheres and suspensions of deformable spheres. In the latter case, the matrix was, again, too compressible $\left(v_{0}=0.38\right)$ and the modulus ratio too low $(\mu=26.83)$. Apart from that, the maximum volume fraction used in their calculations was $\phi=0.5$, which is below the range where the shoulder develops.
When the 2D generalized self-consistent method is used to calculate the relative transverse shear modulus of an elastic fiber-reinforced material, the results show the same dependence on the Poisson ratios and the modulus ratio. Applying the generalized self-consistent method to a suspension of linear viscoelastic materials, similar effects are observed in the storage modulus and the loss modulus.

\section{Comparison to numerical and experimental data}

As explained in the "Nonlinear viscoelastic suspension rheology" section, models for the effects of particles on the linear viscoelastic properties of a suspension, governed by the multipliers $k_{G, i}$ for the modulus and $k_{\lambda, i}$ for the relaxation time, can be validated by numerical simulations. Hwang et al. (2004a) presented results for a sheared $2 \mathrm{D}$ system, consisting of rigid discs suspended in an Oldroyd-B fluid. Their simulation method, based on sliding rectangular domains with periodic boundary conditions, was extended to three dimensions to describe suspensions of spherical particles (Hwang et al. 2004b). It was also modified for 2D extensional flow, based on stretching rectangular domains with periodic boundary conditions (Hwang and Hulsen 2006). An alternative method for 2D extensional flow, using a fixed grid, was developed recently by D'Avino et al. (2007a, b). The 2D shear results are considered here. Figure 5 shows the time-averaged

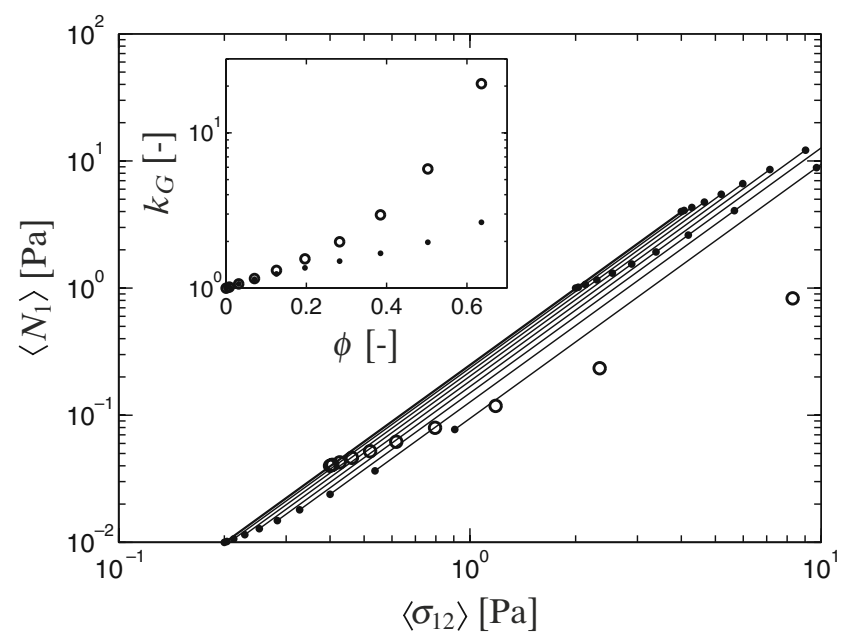

Fig. 5 The steady-state first normal stress difference and shear stress from simulations (lines, Hwang et al. (2004a)) and from the 2D generalized self-consistent method at an arbitrary shear rate (circles). Inset: $k_{G}(\phi)$ from simulations (dots) and from the generalized self-consistent method (circles). The angle brackets indicate that the steady-state properties were obtained from the simulations by averaging over time 
steady-state first normal stress difference $\left\langle N_{1}\right\rangle$ vs the time-averaged steady-state shear stress $\left\langle\sigma_{12}\right\rangle\left(\sigma_{12}\right.$ is the sum of the viscous mode and the viscoelastic mode in the Oldroyd-B model). Each line corresponds to the simulation results at a constant area fraction of disks, which is equivalent to a volume fraction of infinitely long parallel cylinders, and different shear rates.

Because of the rigidity of the particles, $k_{\lambda, i}=1$ and $k_{G, i}=f_{G}$ is a real number, which is obtained from the 2D generalized self-consistent method. Irrespective of the shear rate, shifting $\tau_{12}(\phi=0)$ to the right and $N_{1}(\phi=0)$ upwards by the same factor $f_{G}(\phi)$, we should end up on the line corresponding to the area fraction $\phi$ (see Fig. 1). The circles in Fig. 5 indicate the results of the 2D generalized self-consistent method at an arbitrary constant shear rate. It turns out that these agree with the simulations up to $\phi \approx 0.10$. At larger area fractions, the $2 \mathrm{D}$ generalized self-consistent method predicts a much stronger increase of $f_{G}$ than the simulations. This is not entirely surprising because Hwang et al. (2004a) determined the steady-state suspension properties from simulations with a single particle in a periodic domain. The authors already noted that this method does not give realistic results for highly concentrated systems. Nevertheless, an area fraction of $10 \%$ is quite small.

We also compared the predictions of a single-mode upper convected Maxwell model, combined with the 3D generalized self-consistent method, to the experimental data of Mall-Gleissle et al. (2002). As seen in Fig. 6, the data are underpredicted already for $\phi=0.05$. Better results would probably have been obtained with a more advanced constitutive model. However, even for relatively simple ones, like the Giesekus model or

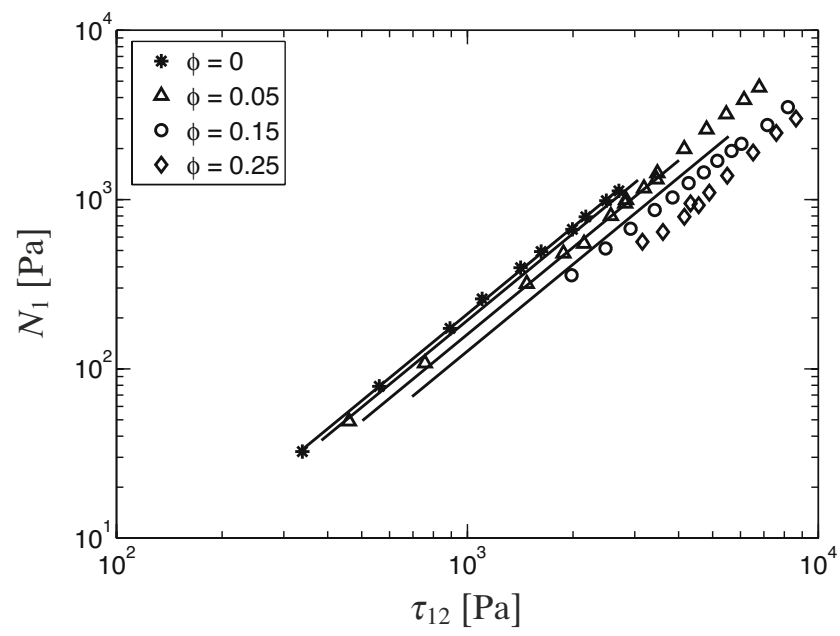

Fig. 6 The steady-state first normal stress difference and shear stress from experiments (symbols, Mall-Gleissle et al. (2002)) and from the 3D generalized self-consistent method (lines) the PTT model, no analytical solutions can be derived for $\tau_{12}$ and $N_{1}$. The relaxation behavior of the matrix has to be known to calculate them numerically. Unfortunately, we do not have this information.

\section{Application to crystallization experiments}

We looked at two types of rheological measurements on crystallizing polymer melts to investigate whether suspension models can indeed capture the phenomena observed in these experiments. In the first type of experiment, after different short periods of shear, the evolution of the linear viscoelastic properties was followed in time at a constant frequency (J.F. Vega, personal communication). In the second type, the linear viscoelastic properties were measured over a range of frequencies for different constant volume fractions of crystallites (Boutahar et al. 1996, 1998; Coppola et al. 2006).

The results from these experiments were used to validate the generalized self-consistent method, as well as the interpolation method, which was described in the "Crystallites as rigid particles" section. While the latter usually treats the highly filled polymer melt as a suspension of amorphous particles in a semicrystalline matrix, the former always takes the crystallites as particles. It has been mentioned in the "History and relation to other models" section that, to allow $\phi \rightarrow 1$, the generalized self-consistent method assumes a broad distribution of particle diameters. This is generally not the case in a crystallizing polymer melt, but there, complete space filling is achieved in a different way. After impingement of the crystallites, further growth will be restricted to the directions in which amorphous material is still present, until all of it has been incorporated in the semicrystalline phase. Formally, because the crystallites become irregularly shaped, the generalized self-consistent method does not apply anymore. However, we do not expect the rheological properties of a highly concentrated suspension to be very sensitive to variations in particle shape.

\section{Evolution of linear viscoelastic properties after short-term shear}

Flow-induced crystallization experiments, carried out in our own group (J.F. Vega, personal communication) are considered first. An isotactic polypropylene melt (HD 120 MO, supplied by Borealis) was subjected to different short periods of shear. Subsequently, its linear viscoelastic properties were monitored in time by means of oscillatory shear measurements. The results are shown in Fig. 7. It is clear that, immediately after 


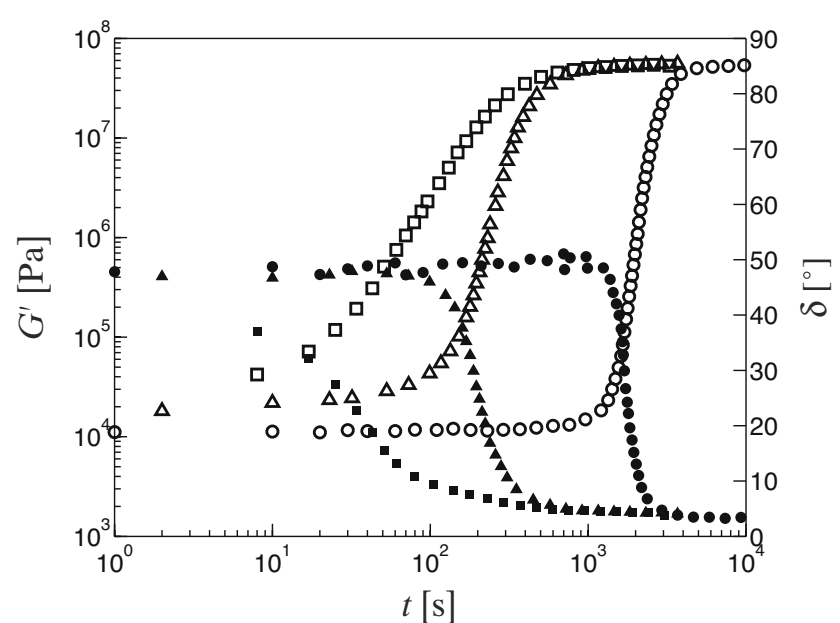

Fig. 7 Evolution of the storage modulus (open symbols) and loss angle (filled symbols) during crystallization, measured under quiescent conditions (open and filled circles) and after shearing at $\dot{\gamma}=60 \mathrm{~s}^{-1}$ for $t_{s}=3 \mathrm{~s}$ (open and filled triangles) and $t_{s}=6 \mathrm{~s}$ (open and filled squares). Part of the data points were omitted for the sake of clarity

the flow, the dynamic modulus of the material, which was then still largely amorphous, was already increased significantly. The first values of $G^{\prime}$ and $G^{\prime \prime}$ measured after the flow were used as $G_{0}^{\prime}$ and $G_{0}^{\prime \prime}$ in the model calculations. The plateaus of $G^{\prime}$ and $\delta$, reached in the late stage of crystallization, were extrapolated to the earlier stages by the functions

$G_{1}^{\prime}(t)= \begin{cases}G_{1}^{\prime}\left(t_{1}\right) & \text { for } t \leq t_{1} \\ G_{1}^{\prime}\left(t_{1}\right)\left[\frac{t}{t_{1}}\right]^{m} & \text { for } t>t_{1}\end{cases}$

and

$\delta_{1}(t)= \begin{cases}\delta_{1}\left(t_{1}\right) & \text { for } t \leq t_{1} \\ \delta_{1}\left(t_{1}\right)+c_{\delta} \ln \left(\frac{t}{t_{1}}\right) & \text { for } t>t_{1}\end{cases}$

and these extrapolations were used as the linear viscoelastic properties of the semicrystalline phase. The values of the parameters in Eqs. 44 and 45 are given in Table 2. The characteristic time $t_{1}$, indicating the transition from the space-filling stage to the perfection stage, was defined as the intersection of the extrapolated linear fits of the $\log \left(G^{\prime}\right)-\log (t)$ data in these stages, as shown in Fig. 8.

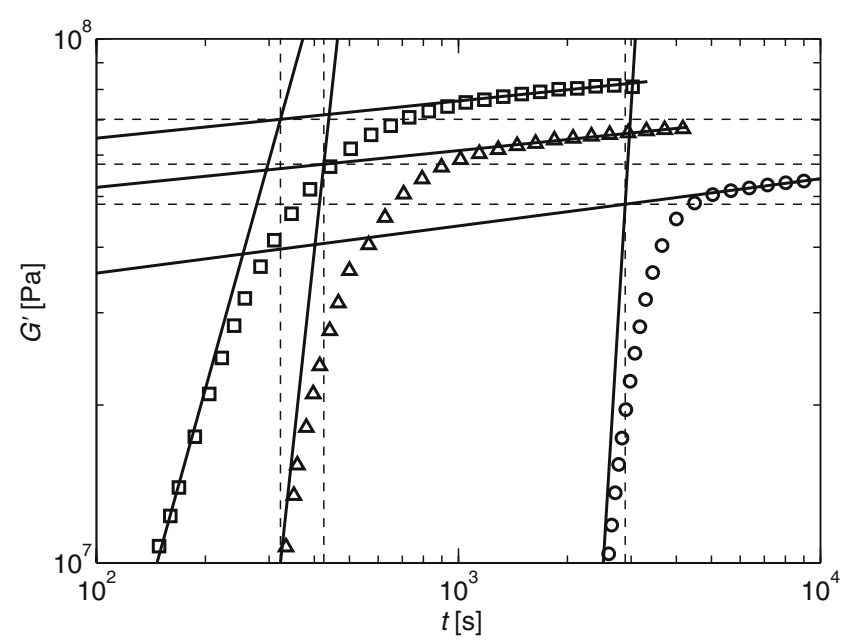

Fig. 8 Close-up of the storage moduli from Fig. 7. Part of the data points were omitted and the curves corresponding to $t_{s}=3 \mathrm{~s}$ and $t_{s}=6 \mathrm{~s}$ were shifted vertically by factors 1.2 and 1.5 , respectively, for the sake of clarity. The solid lines are fits of the data in the plateau region and in the region of strong increase of $G^{\prime}$. The dashed lines indicate $t_{1}$ and $G_{1}^{\prime}\left(t_{1}\right)$

In the experiments with 3 and $6 \mathrm{~s}$ of shear, oriented crystallites were observed. There, the effect of the spherulites was calculated by the 3D generalized selfconsistent method, and the resulting effective rheological properties were used as matrix properties in the 2D generalized self-consistent method. The evolution of the volume fraction of spherulites was determined from optical micrographs taken during the flow and the subsequent crystallization (D.G. Hristova, personal communication). The result for the quiescent melt is shown in Fig. 9. The volume fraction of oriented crystallites could not be determined accurately in this way. Therefore, the degree of crystallinity $\chi$, derived from in situ wide-angle X-ray diffraction (WAXD) measurements (D.G. Hristova, personal communication), was used to estimate the total semicrystalline volume fraction $\phi$. By definition, $\phi$ and $\chi$ are related as

$\phi(t)=\frac{\chi(t)}{\chi_{1}(t)}$.

The integrated intensities $X_{W A X D}$ of the diffraction peaks, which are normalized by the total integrated intensities, are included in Fig. 9 for the quiescent

Table 2 Parameters for calculating the rheological properties and degree of crystallinity of the semicrystalline phase by means of Eqs. 44, 45, and 47

\begin{tabular}{llllllll}
\hline$t_{s}[\mathrm{~s}]$ & $t_{1}[\mathrm{~s}]$ & $G_{1}^{\prime}\left(t_{1}\right)[\mathrm{Pa}]$ & $\delta_{1}\left(t_{1}\right)\left[^{\circ}\right]$ & $\chi_{1}\left(t_{1}\right)[-]$ & $m[-]$ & $c_{\delta}\left[{ }^{\circ}\right]$ & $c_{\chi}\left[\mathrm{s}^{-1}\right]$ \\
\hline 0 & $2.89 \times 10^{3}$ & $4.83 \times 10^{7}$ & 3.48 & - & $9.00 \times 10^{-2}$ & $-1.29 \times 10^{-1}$ & - \\
3 & $4.25 \times 10^{2}$ & $4.80 \times 10^{7}$ & 4.83 & $4.98 \times 10^{-1}$ & $7.02 \times 10^{-2}$ & $-3.40 \times 10^{-1}$ & $6.67 \times 10^{-5}$ \\
6 & $3.22 \times 10^{2}$ & $4.68 \times 10^{7}$ & 4.97 & $5.40 \times 10^{-1}$ & $7.11 \times 10^{-2}$ & $-3.40 \times 10^{-1}$ & 0 \\
\hline
\end{tabular}




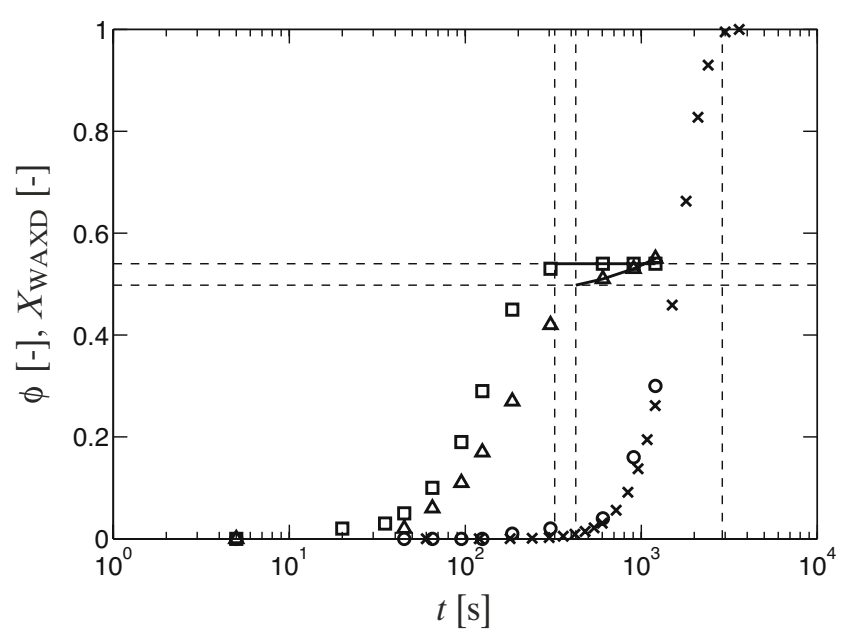

Fig. 9 Space filling during quiescent crystallization, obtained from optical microscopy (crosses), along with the integrated WAXD intensity during quiescent crystallization (circles) and after shearing at $\dot{\gamma}=60 \mathrm{~s}^{-1}$ for $t_{s}=3 \mathrm{~s}$ (triangles) and $t_{s}=6 \mathrm{~s}$ (squares). Solid lines are fits of the WAXD data in the plateau region. Dashed lines indicate $t_{1}$ and $\chi_{1}\left(t_{1}\right)$

and flow-induced crystallization experiments. Comparing the WAXD and optical microscopy results for the quiescent experiment, it is seen that $X_{W A X D}$ is close to but slightly above $\phi$ up to $\phi=0.3$. Because the degree of crystallinity is, by definition, always smaller than the degree of space filling, we conclude that $X_{W A X D}>\chi$. On the other hand, the values of $t_{1}$ derived from the storage modulus measured during flow-induced crystallization (Table 2) correlate well with the onset times of the plateaus in the WAXD data. So at least the time scale is correct, provided that $t_{1}$ corresponds to $\phi=1$ as observed in the quiescent crystallization experiment. We assumed $X_{W A X D}$ to be an adequate measure of the internal degree of crystallinity $\chi=\chi_{1}$ in the plateau region, where the highest signal-to-noise ratio was obtained. Unfortunately, however, the development of $\chi$ and $\chi_{1}$ during the space-filling process could not be reconstructed from these data.

The integrated WAXD intensity in the plateau region was fitted by a linear function of time and extrapolated to obtain $\chi_{1}\left(t>t_{1}\right)$. We assumed that no secondary crystallization took place up to $t=t_{1}$ in any of the experiments considered here, so,

$\chi_{1}(t)=\left\{\begin{array}{ll}\chi_{1}\left(t_{1}\right) & \text { for } t \leq t_{1} \\ \chi_{1}\left(t_{1}\right)+c_{\chi}\left[t-t_{1}\right] & \text { for } t>t_{1}\end{array}\right.$.

Furthermore, for lack of better experimental data, $X_{W A X D}$ was taken as $\chi$ and the experimental data were scaled according to Eqs. 46 and 47 to obtain the total volume fraction. The results are shown in Fig. 10.

The volume fraction of spherulites $\phi_{s p h}$ was determined from optical microscopy in the same way as for the quiescent experiment. The volume fraction of spherulites in the amorphous phase,

$x_{s p h}=\frac{\phi_{s p h}}{1-\phi+\phi_{s p h}}$,

was used in the 3D generalized self-consistent method to calculate the linear viscoelastic properties of the effective matrix in the $2 \mathrm{D}$ generalized self-consistent method. There, the volume fraction of oriented crystallites,

$\phi_{\text {ori }}=\phi-\phi_{s p h}$,

was used to calculate the linear viscoelastic properties of the crystallizing melt.

The results of the generalized self-consistent method are compared to those of the interpolation method in Fig. 11. The main difference is that $G^{\prime}(t)$, according to the generalized self-consistent method, goes through two inflection points before reaching the plateau $G^{\prime}=$ $G_{1}^{\prime}$. This is due to the shoulder in $f_{G}^{*}(\phi)$, as discussed in the "Influence of phase properties" section. The shoulder is followed by a decreasing $G^{\prime}$ for the experiment with $6 \mathrm{~s}$ of shear. This is caused by our estimate of the volume fraction of oriented crystallites, which goes through a maximum and then drops back to zero. The more pronounced local extrema in $\delta$ at large volume fractions (Fig. 12) are caused by local extrema in $G^{\prime \prime}$, which, depending on the phase properties, may arise in the linear viscoelastic generalized self-consistent method. For the interpolation method and the generalized self-consistent method, the data for the highest shear time are not captured. This can be explained by the lack of information about the shape and orientation of particles in the interpolation method and by uncertainties

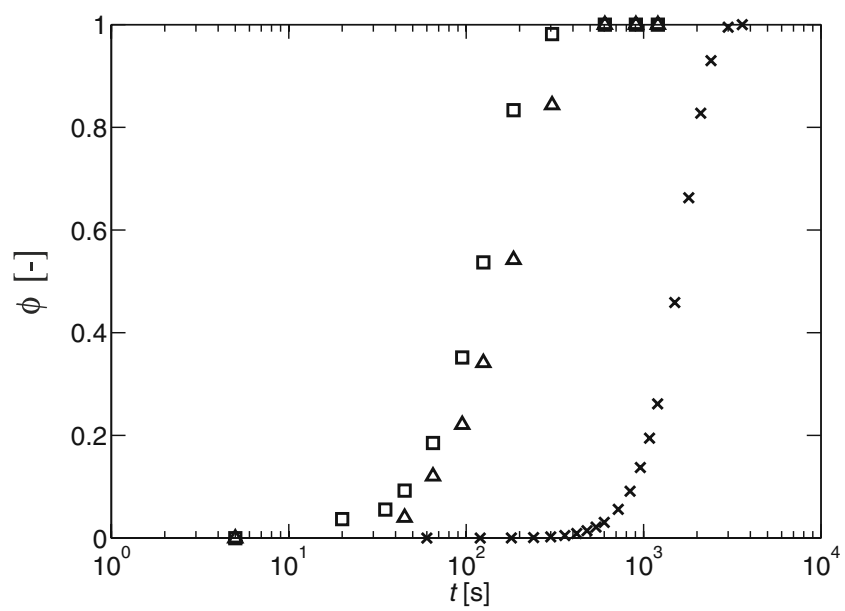

Fig. 10 Space filling, estimated from WAXD for the flowinduced crystallization experiments, as explained in the text 


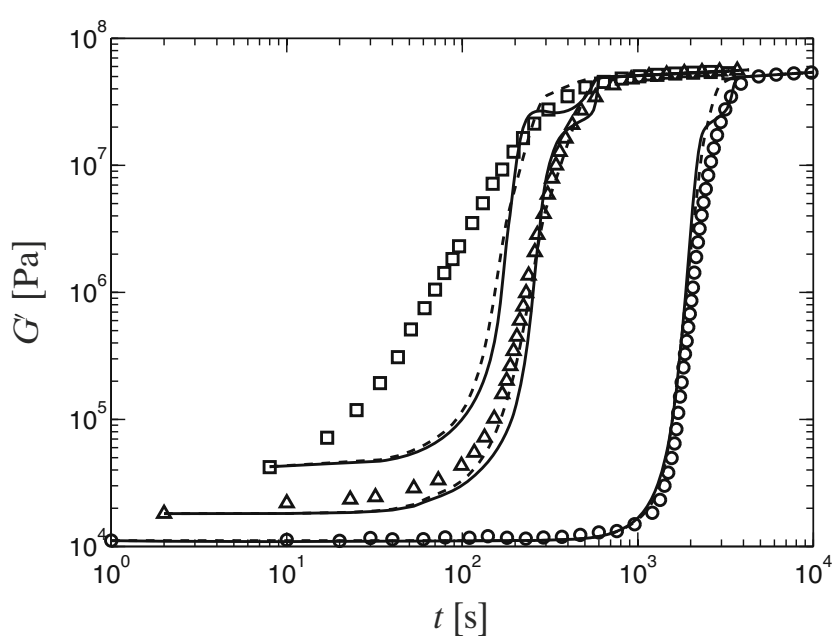

Fig. 11 Evolution of the storage modulus under quiescent conditions (circles) and after shearing at $\dot{\gamma}=60 \mathrm{~s}^{-1}$ for $t_{s}=3 \mathrm{~s}$ (triangles) and $t_{s}=6 \mathrm{~s}$ (squares). Dashed lines were obtained by the interpolation method, Eq. 23, using a hyperbolic tangent function for $w(\phi)$. Solid lines correspond to the generalized self-consistent method, using Eq. 41 with the coefficients given in Table 4 in the Appendix and Eq. 66 from the Appendix

in the estimate of the oriented volume fraction used in the generalized self-consistent method.

The degree of space filling is often estimated by a linear scaling of the storage modulus (Gauthier et al. 1992; Khanna 1993). This can be rewritten as

$G^{\prime}(t)=G_{0}^{\prime}(t)+\left[G_{1}^{\prime}(t)-G_{0}^{\prime}(t)\right] \phi(t)$.

Figure 13 shows that the rheological data are not reproduced from the microscopic images by Eq. 50. Also plotted is the logarithmic scaling law,

$G^{\prime}(t)=G_{0}^{\prime}(t)\left[\frac{G_{1}^{\prime}(t)}{G_{0}^{\prime}(t)}\right]^{\phi(t)}$,

which was used by Pogodina et al. (1999b). It performs much better than the linear scaling law, though not as well as the generalized self-consistent method and the interpolation method.

\section{Frequency sweeps at different volume fractions}

We first consider the experiments of Coppola et al. (2006). They cooled down a poly(1-butene) melt to a temperature $T_{c}$ below the nominal melting temperature $T_{m}$ and let it crystallize at $T_{c}$ for a controlled amount of time. Then, they applied an inverse quench (Acierno and Grizzuti 2003) to a temperature $T_{i q}$ close to, but still below, $T_{m}$ and measured the rheological properties by means of a multiwave technique, which extended the experimentally accessible range of frequencies down to the order of $10^{-3} \mathrm{rad} / \mathrm{s}$. This revealed that the plateau in the storage modulus, observed at

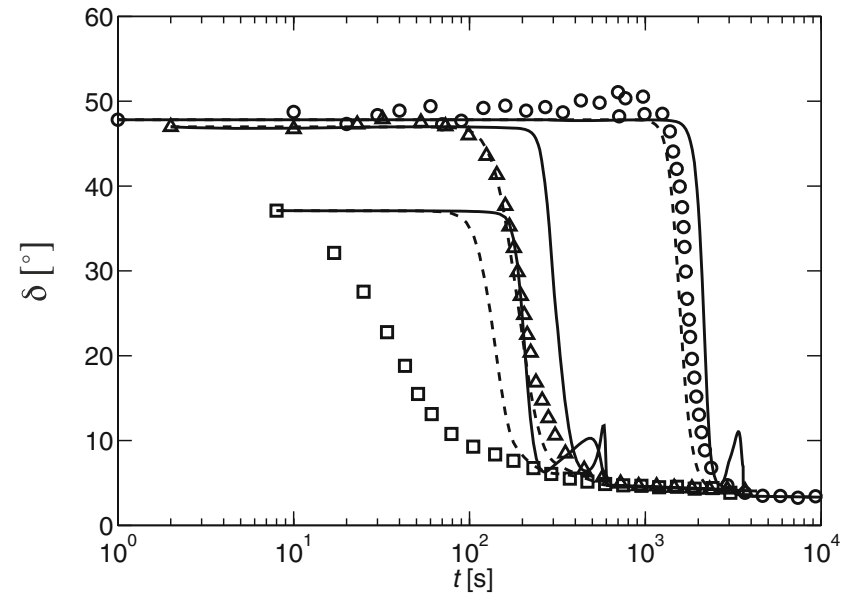

Fig. 12 Evolution of the loss angle, measured under different conditions and calculated by the interpolation method and the generalized self-consistent method, indicated by the symbols and lines as in Fig. 11

low frequencies, did not continue all the way down to zero, as in a chemical or physical gel, but fell off at a certain frequency. This supports the idea that crystallization precursors and/or crystalline nuclei act as physical crosslinks but are too far apart to form a percolating network (Zuidema 2000; Zuidema et al. 2001).

The results of Coppola et al. are reproduced in Fig. 14. Different volume fractions of spherulites were obtained by varying the duration of crystallization at $T_{c}$. The rheological properties of the partially crystallized melts were compared to those of an amorphous melt containing, according to the authors, a much larger volume fraction of solid spheres. The effect observed

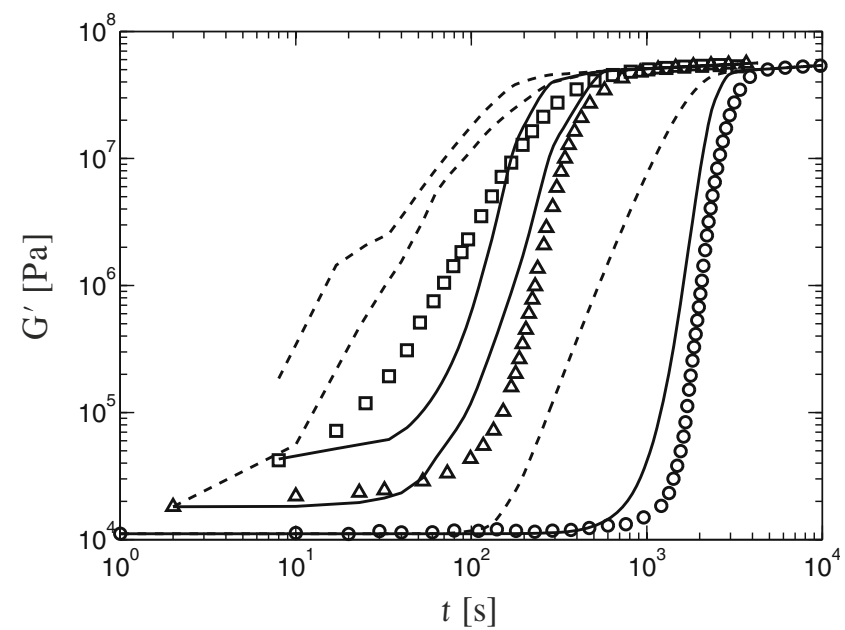

Fig. 13 Evolution of the storage modulus, measured under different conditions, indicated by the symbols and as in Fig. 11. Dashed lines correspond to the linear scaling law, Eq. 50, solid lines to the logarithmic scaling law, Eq. 51 


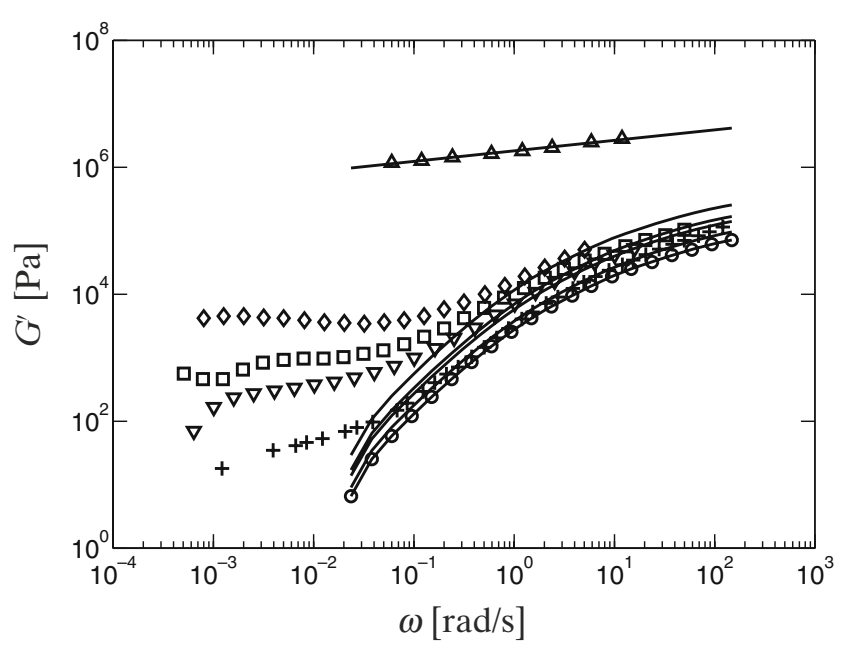

Fig. 14 Storage modulus measured by Coppola et al. (2006) for crystallizing melts (open symbols) and an amorphous melt containing glass beads (crosses). Lines correspond to the 3D generalized self-consistent method

in the low-frequency rheology was much smaller than in the partially crystallized melts, from which the authors concluded that, indeed, the behavior of the latter cannot be explained by gelation. However, they determined the volume fraction by means of the linear scaling law, Eq. 50. Based on the results discussed in the previous section, we believe that the actual volume fraction was much larger and we expect it to be more accurately estimated by the generalized self-consistent method. The results of this model are included in Fig. 14. The low-frequency behavior was obviously not

Table 3 Volume fraction of spherulites, as obtained from the linear scaling law, Eq. $50\left(\phi_{G^{\prime}(t)}\right)$, from differential scanning calorimetry $\left(\phi_{D S C}\right)$, and from the $3 \mathrm{D}$ generalized self-consistent method $\left(\phi_{G S C M}\right)$ for the two sets of experiments

\begin{tabular}{lll}
\hline$\phi_{G S C M}$ & $\phi_{G^{\prime}(t)}$ & $\phi_{D S C}$ \\
\hline Coppola et al. (2006) & & \\
0.25 & 0.0058 & \\
0.30 & 0.0129 & \\
0.40 & 0.0280 & \\
1 & 1 & \\
& $\phi_{\text {beads }}$ & \\
0.12 & 0.12 & \\
Boutahar et al. (1996, 1998) & & 0.1 \\
0.06 & & 0.2 \\
0.11 & & 0.3 \\
0.16 & & 0.4 \\
0.23 & & 0.5 \\
0.32 & & 0.6 \\
0.41 & & 0.7 \\
0.55 & & 0.8 \\
0.73 & &
\end{tabular}

captured for the partially crystallized melts, nor for the particle-filled melt. Therefore, the model was fitted to the data at high frequencies. The volume fractions obtained through both approaches are listed in Table 3 . According to the generalized self-consistent method, the samples were highly filled with spherulites. Unfortunately, no optical data are available to validate this result.

Experiments on two fundamentally different materials were published by Boutahar et al. $(1996,1998)$. One material was a heavily nucleated polyethylene melt, whose morphology looked like that of a colloid. The crystalline nuclei were very small, close together, and highly imperfect. The other was an isotactic polypropylene melt, containing large, well separated spherulites. We will consider the data for this suspension-like material here.

The evolution of the storage modulus and the loss modulus is depicted in Figs. 15 and 16, respectively. From Boutahar et al. (1996), we estimated $G_{1}^{\prime}=10^{7} \mathrm{~Pa}$ and $G_{1}^{\prime \prime}=6 \times 10^{5} \mathrm{~Pa}$. The results are not very sensitive to the values used. Although, at the largest volume fraction, $G^{\prime \prime}$ exceeds $G_{1}^{\prime \prime}$ around $\omega=1 \mathrm{rad} / \mathrm{s}$, the absolute value $\left|G^{*}\right|$ is always smaller than $\left|G_{1}^{*}\right|$, as it should be. The generalized self-consistent method was again fitted to the data at high frequencies. Also for these experiments, the model fails to capture the observed strong increase of the storage and loss moduli with increasing volume fraction at low frequencies. However, the fitted volume fractions correspond very well to the values obtained by Boutahar et al. from differential scanning calorimetry (Table 3).

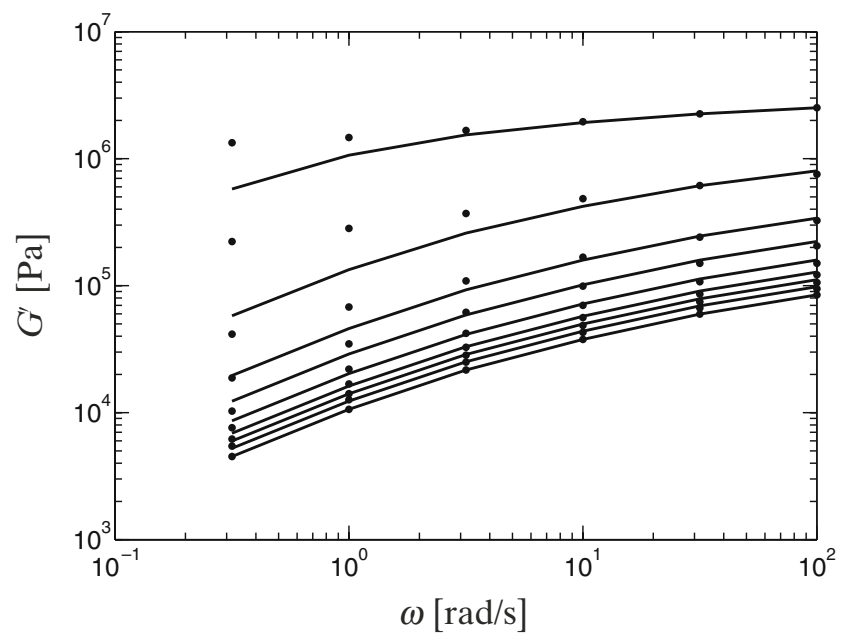

Fig. 15 Storage modulus measured by Boutahar et al. (1996, 1998) (dots) and obtained from the 3D generalized self-consistent method (lines) 


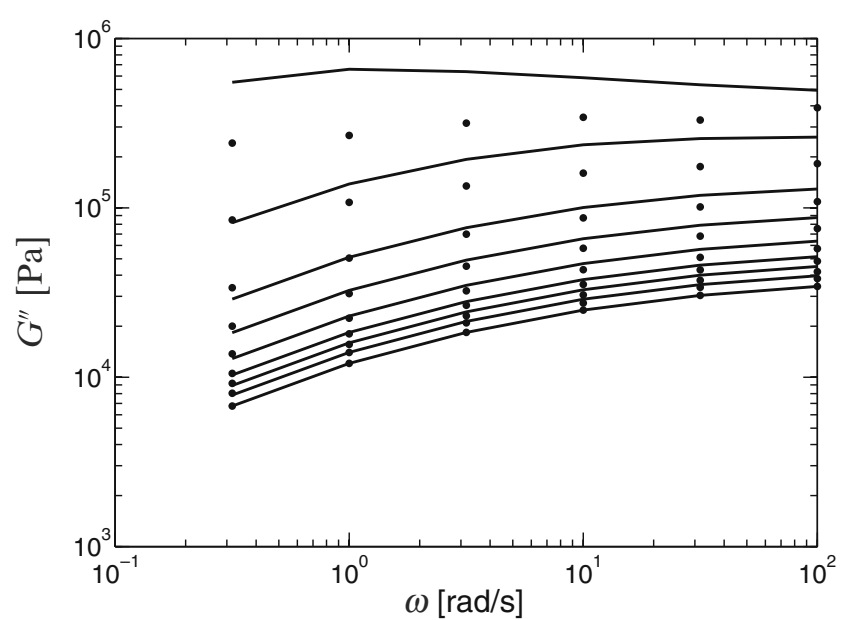

Fig. 16 Loss modulus measured by Boutahar et al. (1996, 1998) (dots) and obtained from the 3D generalized self-consistent method (lines)

\section{Modeling of low-frequency suspension rheology}

A few models describe the development of a lowfrequency plateau in $G^{\prime}$ and $G^{\prime \prime}$. In the Palierne model, it is governed by the surface tension. However, because spherulites behave like solid particles rather than liquid drops, the surface tension is probably negligible in a crystallizing melt, at least for the relatively large volume fractions (corresponding to large radii of spherulites) where the plateau is first observed. Van Ruth et al. (2006) carried out quiescent crystallization experiments, similar to those of Boutahar et al. (1996, 1998). They used the Palierne model with an equilibrium modulus,

$G_{e q}=c\left(\phi-\phi_{0}\right)^{m}$,

added to $G^{\prime}$, which could be fitted to the experimental data with realistic values of the parameters $\phi_{0}$ and $m$. However, the results of the Palierne model for $G^{\prime \prime}$ were not shown. A Cross model was used to fit the dynamic viscosity $\left|\eta^{*}\right|$ for each volume fraction separately.

The self-consistent method predicts a percolation threshold, where the modulus rises or drops steeply, approaching the threshold from below or above, respectively. It diverges to infinity, respectively zero, when both phases are incompressible and the particles are much stiffer than the matrix. This happens at $\phi=0.4$ for spheres (Hill 1965b) and at $\phi=0.5$ for cylindrical fibers (Hill 1965a). When applied to a linear viscoelastic suspension by means of the correspondence principle, the effect is mainly observed at low frequencies as a plateau in $G^{\prime}$. This was shown by Wilbrink et al.
(2005). However, the self-consistent method did not agree quantitatively with their suspensions, for which the percolation threshold was around $\phi=0.10$.

Albérola and Mélé (1996) incorporated the concept of percolation in the generalized self-consistent method. In their modified unit cell, the part of the matrix trapped inside particle clusters is represented by a sphere, which is surrounded by two concentric shells, representing the particles and the nontrapped matrix. This double-coated sphere is surrounded by the effective medium. Hervé and Zaoui $(1993,1995)$ extended the generalized self-consistent method to particles coated by an arbitrary number of layers. Their model was used by Albérola et al. to calculate the rheological properties of a suspension with clustering deformable particles. According to the simulation results of Hwang et al. (2004a, b, 2006), extensional stresses between particles in a sheared viscoelastic matrix enhance their tendency to form clusters. Therefore, we applied the modified generalized self-consistent method to the experiments described in the previous section. However, it turned out that the percolation threshold shifts $f_{G}^{*}$ similarly for all frequencies. Hence, the shoulder, observed experimentally at low frequencies, is not reproduced by this model.

\section{Conclusions}

The approach to suspension-based rheological modeling of crystallizing polymer melts, presented in this paper, has several advantages. Linear viscoelastic suspension models are available to obtain the modulus and relaxation time multipliers $k_{G, i}$ and $k_{\lambda, i}$. These account for the influence of crystallites on the relaxation spectrum used in the constitutive model for the melt. The fact that crystallites can be described as nonrigid particles prevents problems at high degrees of space filling. The phenomenological nonlinear viscoelastic suspension model qualitatively reproduces results of numerical simulations but is much less expensive computationally and can therefore easily be used in industrial processing simulations. Only standard rheological experiments are needed to quantify the phenomenological parameter $k_{L}$.

After considering several linear viscoelastic suspension models, we used the generalized self-consistent method (Christensen 1990; Christensen and Lo 1979, 1986) because of its simplicity and because, in combination with the nonlinear viscoelastic model, it satisfies all the requirements stated in the Introduction. Combined 
rheometry and optical microscopy showed that a widely used linear scaling of the storage modulus (Gauthier et al. 1992; Khanna 1993) severely underpredicted the degree of space filling. The generalized self-consistent method described the evolution of linear viscoelastic properties during crystallization rather well, at least at moderate to high frequencies; the low-frequency behavior (Boutahar et al. 1996, 1998; Coppola et al. 2006) was not captured. An extension of the generalized selfconsistent method, including percolation (Albérola and Mélé 1996), did not solve this problem; the relative dynamic modulus was merely shifted in a similar manner for all frequencies.

Acknowledgements We are grateful to Dr. J.F. Vega (Consejo Superior de Investigaciones Cientificas Madrid, Spain) and Dr. D.G. Hristova (TU/e, The Netherlands) for providing some of their experimental results.

Open Access This article is distributed under the terms of the Creative Commons Attribution Noncommercial License which permits any noncommercial use, distribution, and reproduction in any medium, provided the original author(s) and source are credited.

\section{Appendix}

$3 D$ generalized self-consistent method and Bousmina's linear viscoelastic model For the 3D generalized selfconsistent method, the coefficients are summarized in Table 4. Some of the expressions given by Bousmina (1999) as first-order approximations of $A, B$, and $C$ contain errors, most of which are due to misprints in Christensen and Lo (1979). Bousmina's Eqs. 54-59 should read

$$
\begin{aligned}
A= & K_{1}\left[24 K_{2}-150 K_{3} \phi\right], \\
B= & \frac{1}{2} K_{1}\left[9 K_{2}+375 K_{3} \phi\right], \\
C= & \frac{1}{4} K_{1}\left[-114 K_{2}-675 K_{3} \phi\right], \\
K_{1}= & {\left[\frac{5}{2}\left(\frac{G_{1}^{*}+\alpha / R}{G_{0}^{*}}-8\right)\right.} \\
& \left.+7\left(\frac{G_{1}^{*}+\alpha / R}{G_{0}^{*}}\right)+4\right], \\
K_{2}= & \left(\frac{G_{1}^{*}+\alpha / R}{G_{0}^{*}}+\frac{3}{2}\right), \\
K_{3}= & \left(\frac{G_{1}^{*}+\alpha / R}{G_{0}^{*}}-1\right) .
\end{aligned}
$$

$2 D$ generalized self-consistent method Under the assumption of transverse isotropy, Hooke's law for an elastic fiber suspension can be written in matrix form according to

$$
\left[\begin{array}{c}
\tau_{11} \\
\tau_{22} \\
\tau_{33} \\
\tau_{12} \\
\tau_{13} \\
\tau_{23}
\end{array}\right]=\left[\begin{array}{cccccc}
C_{11} & C_{12} & C_{13} & 0 & 0 & 0 \\
C_{12} & C_{22} & C_{23} & 0 & 0 & 0 \\
C_{13} & C_{23} & C_{22} & 0 & 0 & 0 \\
0 & 0 & 0 & C_{44} & 0 & 0 \\
0 & 0 & 0 & 0 & C_{44} & 0 \\
0 & 0 & 0 & 0 & 0 & C_{22}-C_{23}
\end{array}\right]\left[\begin{array}{c}
\epsilon_{11} \\
\epsilon_{22} \\
\epsilon_{33} \\
2 \epsilon_{12} \\
2 \epsilon_{13} \\
2 \epsilon_{23}
\end{array}\right]
$$

Table 4 Parameters used in the 3D generalized self-consistent method (Christensen and Lo 1979, 1986; Christensen 1990)

Shear modulus $G$, Eq. 40

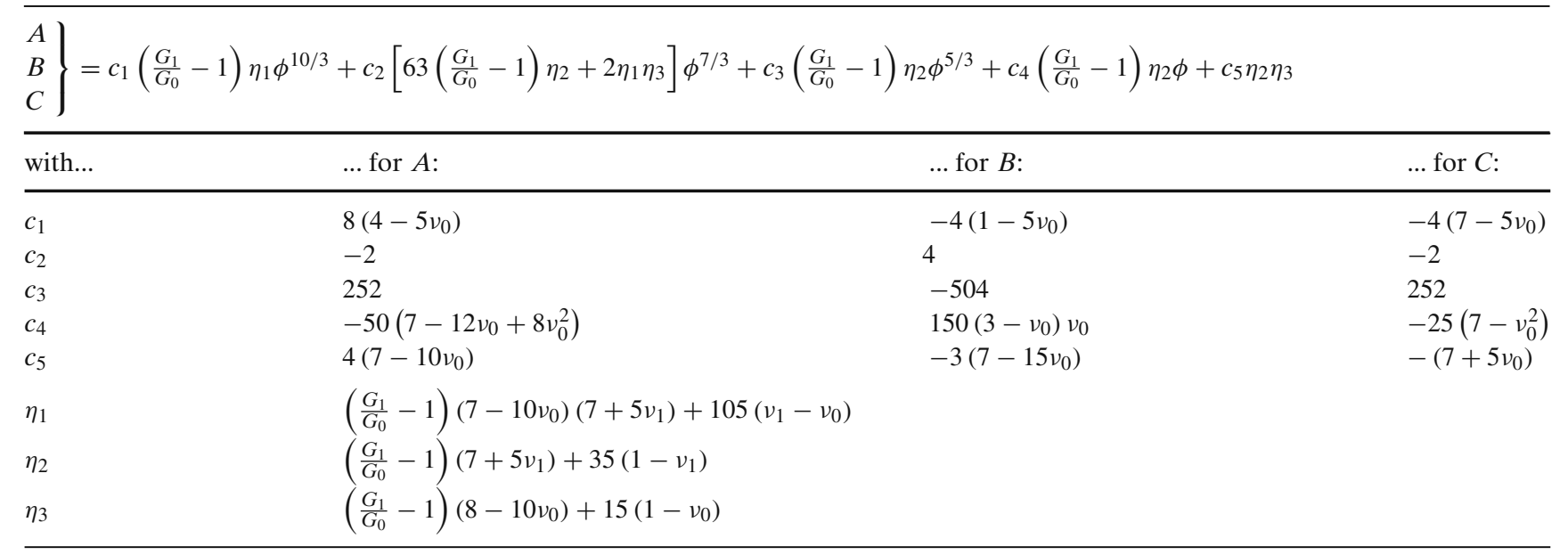


Table 5 Parameters used in the composite cylinders model (Hashin and Rosen 1964) and the 2D generalized self-consistent method (Christensen and Lo 1979, 1986; Christensen 1990)

\begin{tabular}{lc}
\hline Longitudinal Young's modulus & $E_{11}$, Eq. 68 \\
$D_{1}$ & $1-v_{1}$ \\
$D_{2}$ & $\frac{1+\phi}{1-\phi}+v_{0}$ \\
$D_{3}$ & $2 v_{1}^{2}$ \\
Poisson ratio $v_{12}$, Eq. 70 & \\
$L_{1}$ & $2 v_{1}\left(1-v_{0}^{2}\right) \phi+v_{0}\left(1+v_{0}\right)(1-\phi)$ \\
$L_{2}$ & $\left(1-v_{1}-2 v_{1}^{2}\right) \phi$ \\
$L_{3}$ & $2\left(1-v_{0}^{2}\right) \phi+\left(1+v_{0}\right)(1-\phi)$
\end{tabular}

Transverse shear modulus $G_{23}$, Eq. 40

\begin{tabular}{lccc}
$A$ & $3 c_{1} c_{2} \phi(1-\phi)^{2}+\left(c_{2} \eta_{0}-c_{3} \phi^{3}\right)\left(c_{1} \eta_{0} \phi-c_{4}\right)$ \\
$B$ & $-6 c_{1} c_{2} \phi(1-\phi)^{2}+\left[c_{2}\left(\eta_{0}-1\right)-2 c_{3} \phi^{3}\right]\left(c_{1} \phi+c_{4}\right)$ & \\
$C$ & $3 c_{1} c_{2} \phi(1-\phi)^{2}+\left(c_{2}+c_{3} \phi^{3}\right)\left(c_{1} \phi+c_{4}\right)$ & $\eta_{0}$ & $\eta_{1}$ \\
$c_{1}$ & $c_{2}$ & $\frac{G_{1}}{G_{0}} \eta_{0}+1$ & $3-4 v_{0}$ \\
$\frac{G_{1}}{G_{0}}-1$ & $\frac{G_{1}}{G_{0}}+\eta_{1}$ & $\frac{G_{1}}{G_{0}} \eta_{0}-\eta_{1}$ & $3-4 v_{1}$ \\
\hline
\end{tabular}

with the index 1 corresponding to the direction of the fiber axes and the indices 2 and 3 corresponding to perpendicular directions in the transverse plane. Hashin and Rosen (1964) conveniently selected the following moduli to describe the mechanical behavior of the suspension: the plane-strain bulk modulus

$K_{23}=\frac{C_{22}+C_{23}}{2}$,

the transverse shear modulus

$G_{23}=\frac{C_{22}-C_{23}}{2}$,

the longitudinal shear modulus

$G_{12}=G_{13}=C_{44}$,

the longitudinal Young's modulus

$E_{11}=C_{11}-\frac{2 C_{12}^{2}}{C_{22}+C_{23}}$,

and $C_{11}$. In the case of a random arrangement of the fibers across the 23-plane, they found

$\frac{K_{23}}{K_{0}}=\frac{\left(1+2 v_{0} \phi\right) \frac{K_{1}}{K_{0}}+2 v_{0}(1-\phi)}{(1-\phi) \frac{K_{1}}{K_{0}}+2 v_{0}+\phi}$,

$$
\begin{aligned}
\frac{G_{12}}{G_{0}}= & \frac{(1+\phi) \frac{G_{1}}{G_{0}}+1-\phi}{(1-\phi) \frac{G_{1}}{G_{0}}+1+\phi}, \\
\frac{E_{11}}{E_{0}}= & {\left[\left(\frac{E_{1}}{E_{0}}-1\right) \phi+1\right] } \\
& \times\left[\frac{D_{1}-D_{3} F_{1}+\left(D_{2}-D_{4} F_{2}\right) \frac{E_{1}}{E_{0}}}{D_{1}-D_{3}+\left(D_{2}-D_{4}\right) \frac{E_{1}}{E_{0}}}\right],
\end{aligned}
$$

and

$C_{11}=E_{11}+4 v_{12}^{2} K_{23}$,

where

$v_{12}=v_{13}=\frac{L_{1} \phi \frac{E_{1}}{E_{0}}+L_{2} v_{0}(1-\phi)}{L_{3} \phi \frac{E_{1}}{E_{0}}+L_{2}(1-\phi)}$

is the Poisson ratio for uniaxial stress in the direction of the fiber axes. The parameters $D_{1}, D_{2}, D_{3}, D_{4}, F_{1}$, $F_{2}, L_{1}, L_{2}$, and $L_{3}$ depend on the properties of the individual phases and on $\phi$ and are given in Table 5. The same effective properties follow from the $2 \mathrm{D}$ generalized self-consistent method, which additionally gives the relative transverse shear modulus $G_{23} / G_{0}$ from Eq. 40 with the parameters $A, B$, and $C$ from Table 5. 


\section{References}

Acierno S, Grizzuti N (2003) Measurements of the rheological behavior of a crystallizing polymer by an "inverse quenching" technique. J Rheol 47:563-576

Agbossou A, Bergeret A, Benzarti K, Albérola N (1993) Modelling of the viscoelastic behaviour of amorphous thermoplastic/glass beads composites based on the evaluation of the complex Poisson's ratio of the polymer matrix. J Mater Sci 28:1963-1972

Albérola ND, Mélé P (1996) Viscoelasticity of polymers filled by rigid or soft particles: theory and experiment. Polym Compos 17:751-759

Aral BK, Kalyon DM (1997) Viscoelastic material functions of noncolloidal suspensions with spherical particles. J Rheol 41:599-620

Benveniste Y (1987) A new approach to the application of MoriTanaka's theory in composite materials. Mech Mater 6: $147-157$

Beris AN, Edwards BJ (1994) Thermodynamics of flowing systems with internal microstructure. Oxford University Press, Oxford

Bousmina M (1999) Rheology of polymer blends: linear model for viscoelastic emulsions. Rheol Acta 38:73-83

Boutahar K, Carrot C, Guillet J (1996) Polypropylene during crystallization from the melt as a model for the rheology of molten-filled polymers. J Appl Polym Sci 60:103-114

Boutahar K, Carrot C, Guillet J (1998) Crystallization of polyolefins from rheological measurements-relation between the transformed fraction and the dynamic moduli. Macromolecules 31:1921-1929

Bruggeman DAG (1935) Berechnung verschiedener physikalischer Konstanten von heterogenen Substanzen I. Dielektrizitätskonstanten und Leitfähigkeiten der Mischkörper aus isotropen Substanzen. Ann Phys Folge 5 24: 636-679

Budiansky B (1965) On the elastic moduli of some heterogeneous materials. J Mech Phys Solids 13:223-227

Christensen RM (1969) Viscoelastic properties of heterogeneous media. J Mech Phys Solids 17:23-41

Christensen RM (1990) A critical evaluation for a class of micromechanics models. J Mech Phys Solids 38:379-404

Christensen RM, Lo KH (1979) Solutions for effective shear properties in three phase sphere and cylinder models. J Mech Phys Solids 27:315-330

Christensen RM, Lo KH (1986) Erratum: solutions for effective shear properties in three phase sphere and cylinder models. J Mech Phys Solids 34:639

Coppola S, Acierno S, Grizzuti N, Vlassopoulos D (2006) Viscoelastic behavior of semicrystalline thermoplastic polymers during the early stages of crystallization. Macromolecules 39:1507-1514

D'Avino G, Maffettone PL, Hulsen MA, Peters GWM (2007a) A numerical method for simulating concentrated rigid particle suspensions in an elongational flow using a fixed grid. J Comp Phys 226:688-711

D'Avino G, Maffettone PL, Hulsen MA, Peters GWM (2007b) Numerical simulation of planar elongational flow of concentrated rigid particle suspensions in a viscoelastic fluid. $\mathrm{J}$ Non-Newton Fluid Mech 150:65-79

Doufas AK, McHugh AJ (2001a) Simulation of melt spinning including flow-induced crystallization. Part III: quantitative comparisons with PET spinline data. J Rheol 45: $403-420$
Doufas AK, McHugh AJ (2001b) Two-dimensional simulation of melt spinning with a microstructural model for flow-induced crystallization. J Rheol 45:855-879

Doufas AK, McHugh AJ (2001c) Simulation of film blowing including flow-induced crystallization. J Rheol 45:1085-1104

Doufas AK, Dairanieh IS, McHugh AJ (1999) A continuum model for flow-induced crystallization of polymer melts. J Rheol 43:85-109

Doufas AK, McHugh AJ, Miller C (2000a) Simulation of melt spinning including flow-induced crystallization. Part I: model development and predictions. J Non-Newton Fluid Mech 92:27-66

Doufas AK, McHugh AJ, Miller C, Immaneni A (2000b) Simulation of melt spinning including flow-induced crystallization. Part II: quantitative comparisons with industrial spinline data. J Non-Newton Fluid Mech 92:81-103

Frankel NA, Acrivos A (1967) On the viscosity of a concentrated suspension of solid spheres. Chem Eng Sci 22:847-853

Fröhlich H, Sack R (1946) Theory of the rheological properties of dispersions. Proc R Soc Lond A 185:415-430

Gauthier C, Chailan JF, Chauchard J (1992) Utilisation de l'analyse viscoélastique dynamique à l'étude de la crystallisation isotherme du poly (téréphtalate d'ethylène) amorphe. Application à des composites unidirectionnels avec fibres de verre. Makromol Chem 193:1001-1009

Graebling D, Muller R, Palierne JF (1993) Linear viscoelastic behavior of some incompatible polymer blends in the melt. Interpretation of data with a model of emulsion of viscoelastic liquids. Macromolecules 26:320-329

Grmela M, Öttinger HC (1997) Dynamics and thermodynamics of complex fluids. I. Development of a general formalism. Phys Rev E 56:6620-6632

Hashin Z (1962) The elastic moduli of heterogeneous materials. Trans ASME E84. J Appl Mech 29:143-150

Hashin Z (1965) Viscoelastic behavior of heterogeneous media. Trans ASME E87. J Appl Mech 32:630-636

Hashin Z (1970a) Complex moduli of viscoelastic compositesI. General theory and application to particulate composites. Int J Solids Struct 6:539-552

Hashin Z (1970b) Complex moduli of viscoelastic compositesII. Fiber reinforced materials. Int J Solids Struct 6:797-807

Hashin Z, Shtrikman S (1963) A variational approach to the theory of the elastic behaviour of multiphase materials. J Mech Phys Solids 11:127-140

Hashin Z, Rosen BW (1964) The elastic moduli of fiberreinforced materials. Trans ASME E86. J Appl Mech $31: 223-232$

Hermans JJ (1967) The elastic properties of fiber reinforced materials when the fibers are aligned. Proc K Ned Akad Wet B 70:1-9

Hervé E, Zaoui A (1993) n-Layered inclusion-based micromechanical modelling. Int J Eng Sci 31:1-10

Hervé E, Zaoui A (1995) Elastic behaviour of multiply coated fibre-reinforced composites. Int J Eng Sci 33:1419-1433

Hill R (1965a) Theory of mechanical properties of fibrestrengthened materials: III. Self-consistent model. J Mech Phys Solids 13:189-198

Hill R (1965b) A self-consistent mechanics of composite materials. J Mech Phys Solids 13:213-222

Horst RH, Winter HH (2000a) Stable critical Gels of a crystallizing copolymer of ethene and 1-butene. Macromolecules 33:130-136

Horst RH, Winter HH (2000b) Stable critical gels of a copolymer of ethene and 1-butene achieved by partial melting and recrystallization. Macromolecules 33:7538-7543 
Hütter M (2001) Thermodynamically consistent incorporation of the Schneider rate equations into two-phase models. Phys Rev E 64:011209-1-011209-11

Hütter M, Rutledge GC, Armstrong RC (2005) Crystal shapes and crystallization in continuum modeling. Phys Fluids 17:014107-1-014107-13

Hwang WR, Hulsen MA (2006) Direct numerical simulations of hard particle suspensions in planar elongational flow. J NonNewton Fluid Mech 136:167-178

Hwang WR, Hulsen MA, Meijer HEH (2004a) Direct simulations of particle suspensions in a viscoelastic fluid in sliding bi-periodic frames. J Non-Newton Fluid Mech 121:15-33

Hwang WR, Hulsen MA, Meijer HEH, Kwon TH (2004b) Direct numerical simulations of suspensions of spherical particles in a viscoelastic fluid in sliding tri-periodic domains. In: Proc $\mathrm{XIV}^{\text {th }}$ Int Congr Rheol, Seoul, 22-27 August 2004

Hwang WR, Peters GWM, Hulsen MA, Meijer HEH (2006) Modeling of flow-induced crystallization of particle-filled polymers. Macromolecules 39:8389-8398

Janeschitz-Kriegl H (2003) How to understand nucleation in crystallizing polymer melts under real processing conditions. Colloid Polym Sci 281:1157-1171

Janeschitz-Kriegl H, Ratajski E, Stadlbauer M (2003) Flow as an effective promotor of nucleation in polymer melts: A quantitative evaluation. Rheol Acta 42:355-364

Janeschitz-Kriegl H, Ratajski E (2005) Kinetics of polymer crystallization under processing conditions: transformation of dormant nuclei by the action of flow. Polymer 46:3856-3870

Kerner EH (1956) The elastic and thermo-elastic properties of composite media. Proc Phys Soc Lond B 69:808-813

Khanna YP (1993) Rheological mechanism and overview of nucleated crystallization kinetics. Macromolecules 26:3639-3643

Larson RG (1988) Constitutive equations for polymer melts and solutions. Butterworth, London

Liedauer S, Eder G, Janeschitz-Kriegl H, Jerschow P, Geymayer W, Ingolic E (1993) On the kinetics of shear induced crystallization in polypropylene. Int Polym Process 8:236-244

Likhtman AE, Graham RS (2003) Simple constitutive equation for linear polymer melts derived from molecular theory: Rolie-Poly equation. J Non-Newton Fluid Mech 114:1-12

Mall-Gleissle SE, Gleissle W, McKinley GH, Buggisch H (2002) The normal stress behavior of suspensions with viscoelastic matrix fluids. Rheol Acta 41:61-76

McLeish TCB, Larson RG (1998) Molecular constitutive equations for a class of branched polymers: the pom-pom polymer. J Rheol 42:81-110

Nemat-Nasser S, Yu N (1993) Bounds and estimates of overall moduli of composites with periodic microstructure. Mech Mater 15:163-181

Nemat-Nasser S, Hori M (1993) Micromechanics: overall properties of heterogeneous materials. Elsevier, Amsterdam

Ohl N, Gleissle W (1993) The characterization of the steady-state shear and normal stress functions of highly concentrated suspensions formulated with viscoelastic liquids. J Rheol 37:381-406

Oldroyd JG (1953) The elastic and viscous properties of emulsions and suspensions. Proc R Soc Lond A 218:122-132

Öttinger HC, Grmela M (1997) Dynamics and thermodynamics of complex fluids. II. Illustrations of a general formalism. Phys Rev E 56:6633-6655

Palierne JF (1990) Linear rheology of viscoelastic emulsions with interfacial tension. Rheol Acta 29:204-214 (errata available at http://www.mxpl.net)

Peters GWM, Baaijens FPT (1997) Modelling of non-isothermal viscoelastic flows. J Non-Newton Fluid Mech 68:205-224
Phan-Thien N, Pham DC (1997) Differential multiphase models for polydispersed suspensions and particulate solids. J NonNewton Fluid Mech 72:305-318

Pogodina NV, Winter HH (1998) Polypropylene crystallization as a physical gelation process. Macromolecules 31:8164-8172

Pogodina NV, Siddiquee SK, Van Egmond JW, Winter HH (1999a) Correlation of rheology and light scattering in isotactic polypropylene during early stages of crystallization. Macromolecules 32:1167-1174

Pogodina NV, Winter HH, Srinivas S (1999b) Strain effects on physical gelation of crystallizing isotactic polypropylene. J Polym Sci B Polym Phys 37:3512-3519

Pogodina NV, Lavrenko VP, Srinivas S, Winter HH (2001) Rheology and structure of isotactic polypropylene near the gel point: quiescent and shear-induced crystallization. Macromolecules 42:9031-9043

Schaink HM, Slot JJM, Jongschaap RJJ, Mellema J (2000) The rheology of systems containing rigid spheres suspended in both viscous and viscoelastic media, studied by Stokesian dynamics simulations. J Rheol 44:473-498

Schneider W, Köppl A, Berger J (1988) Non-isothermal crystallization of polymers. System of rate equations. Int Polym Process 2:151-154

See H, Jiang P, Phan-Thien N (2000) Concentration dependence of the linear viscoelastic properties of particle suspensions. Rheol Acta 39:131-137

Segurado J, Llorca J (2002) A numerical approximation to the elastic properties of sphere-reinforced composites. J Mech Phys Solids 50:2107-2121

Tanner RI (2002) A suspension model for low shear rate polymer solidification. J Non-Newton Fluid Mech 102:397-408

Tanner RI (2003) On the flow of crystallizing polymers I. Linear regime. J Non-Newton Fluid Mech 112:251-268

Tanner RI, Qi F (2005) A phenomenological approach to suspensions with viscoelastic matrices. Korea-Austr Rheol J 17:149-156

Thomas DG (1965) Transport characteristics of suspension: VIII. A note on the viscosity of Newtonian suspensions of uniform spherical particles. J Colloid Sci 20:267-277

Torquato S (1997) Effective stiffness tensor of composite media-I. Exact series expansions. J Mech Phys Solids 45: $1421-1448$

Torquato S (1998) Effective stiffness tensor of composite media: II. Applications to isotropic dispersions. J Mech Phys Solids 46:1411-1440

Torquato S (2002) Random heterogeneous materials: microstructure and macroscopic properties. Springer, Berlin Heidelberg New York

Uemura S, Takayanagi M (1966) Application of the theory of elasticity and viscosity of two-phase systems to polymer blends. J Appl Polym Sci 10:113-125

Van Meerveld J (2005) Model development and validation of rheological and flow induced crystallization models for entangled polymer melts. Ph.D. thesis, Eidgenössische Technische Hochschule Zürich (available at www.polyphys. mat.ethz.ch)

Van Meerveld J, Hütter M, Peters GWM (2008) Continuum model for the simulation of fiber spinning, with quiescent and flow induced crystallization. J Non-Newton Fluid Mech 150:177-195

Van Ruth NJL, Vega JF, Rastogi S, Martínez-Salazar J (2006) Viscoelastic behaviour during the crystallisation of isotactic polypropylene. J Mater Sci 41:3899-3905

Verbeeten WMH, Peters GWM, Baaijens FPT (2004) Numerical simulations of the planar contraction flow for a poly- 
ethylene melt using the XPP model. J Non-Newton Fluid Mech 117:73-84

Walpole LJ (1966) On bounds for the overall elastic moduli of inhomogeneous systems - I. J Mech Phys Solids 14: $151-162$

Wassner E, Maier RD (2000) Shear-induced crystallization of polypropylene melts. In: Proc XIII ${ }^{\text {th }}$ International Congress on Rheology, Cambridge, 20-25 August 2000

Waterman HA (1977) Relations between loss angles in isotropic linear viscoelastic materials. Rheol Acta 16:31-42

Wilbrink MWL, Michels MAJ, Vellinga WP, Meijer HEH (2005) Rigidity percolation in dispersions with a structured viscoelastic matrix. Phys Rev E 71:031402-1031402-10

Winter HH, Mours M (1997) Rheology of polymers near liquidsolid transitions. Adv Polym Sci 134:165-234

Zuidema H (2000) Flow-induced crystallization of polymers; application to injection moulding. Ph.D. thesis, Technische Universiteit Eindhoven (available at www.mate.tue.nl/ mate)

Zuidema H, Peters GWM, Meijer HEH (2001) Development and validation of a recoverable strain-based model for flowinduced crystallization of polymers. Macromol Theory Simul 10:447-460 